Geometry $\&$ Topology

Volume 5 (2001) 719-760

Published: 11 October 2001

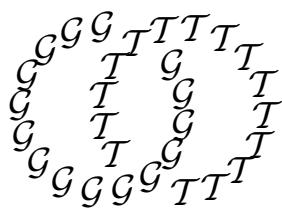

\title{
Generating function polynomials for legendrian links
}

\author{
LisA TRAYNOR \\ Mathematics Department, Bryn Mawr College \\ Bryn Mawr, PA 19010, USA \\ Email: ltraynor@brynmawr.edu
}

\begin{abstract}
It is shown that, in the 1-jet space of the circle, the swapping and the flyping procedures, which produce topologically equivalent links, can produce nonequivalent legendrian links. Each component of the links considered is legendrian isotopic to the 1 -jet of the 0 -function, and thus cannot be distinguished by the classical rotation number or Thurston-Bennequin invariants. The links are distinguished by calculating invariant polynomials defined via homology groups associated to the links through the theory of generating functions. The many calculations of these generating function polynomials support the belief that these polynomials carry the same information as a refined version of Chekanov's first order polynomials which are defined via the theory of holomorphic curves.
\end{abstract}

AMS Classification numbers Primary: 53D35

Secondary: 58E05

Keywords: Contact topology, contact homology, generating functions, legendrian links, knot polynomials

Proposed: Yasha Elaishberg

Seconded: Joan Birman, Robion Kirby

Received: 15 June 2001

Revised: 6 September 2001

(C) Geometry $\mathcal{E}$ Topology Publications 


\section{Introduction}

The 1-jet space of the circle, $\mathcal{J}^{1}\left(S^{1}\right)$, is a manifold diffeomorphic to $S^{1} \times \mathbb{R}^{2}$ :

$$
\mathcal{J}^{1}\left(S^{1}\right)=T^{*}\left(S^{1}\right) \times \mathbb{R}=\left\{(q, p, z): q \in S^{1}, p, z \in \mathbb{R}\right\} .
$$

Viewing $S^{1}$ as a quotient of the unit interval, $S^{1}=\{q \in[0,1]: 0 \sim 1\}$, it is easy to visualize knots in $\mathcal{J}^{1}\left(S^{1}\right)$ as quotients of arcs in $I \times \mathbb{R}^{2}$. This paper focuses on two-component links in $\mathcal{J}^{1}\left(S^{1}\right)$ that satisfy a geometrical condition imposed by the standard contact structure. This standard contact structure on $\mathcal{J}^{1}\left(S^{1}\right)$ is a field of hyperplanes $\xi$ given by $\xi=\operatorname{ker}(d z-p d q)$. There are no integral surfaces of this hyperplane distribution; however, there are integral curves. An immersed curve $\mathcal{L}$ in $\left(\mathcal{J}^{1}\left(S^{1}\right), \xi\right)$ is legendrian if it is tangent to $\xi, T \mathcal{L} \subset \xi$. Functions on $S^{1}$ with values in $\mathbb{R}$ give rise to legendrian knots in $\left(\mathcal{J}^{1}\left(S^{1}\right), \xi\right)$ : the graph of a smooth 1 -periodic function $f$ in the $(q, z)$-plane has a lift to an embedded legendrian curve $j^{1}(f):=\left\{(q, p, z): z=f(q), p=\frac{d}{d q} f(q)\right\}$. Notice that $j^{1}(f)$ is the 1 -jet of the function $f$. Similarly, the graphs of two smooth functions $f, g: S^{1} \rightarrow \mathbb{R}$ will lift to a legendrian link as long as they have no points of tangency. In [19], a link of the form $j^{1}(f) \amalg j^{1}(g)$ is considered where $f, g: S^{1} \rightarrow \mathbb{R}$ satisfy $f(q)>0$ and $g(q)<0$, for all $q \in S^{1}$. In particular, it is shown that $j^{1}(f) \amalg j^{1}(g)$ is an "ordered" legendrian link. A legendrian link $\Lambda_{1} \amalg \Lambda_{0}$ is ordered if $\Lambda_{1} \amalg \Lambda_{0}$ is not legendrianly equivalent to $\Lambda_{0} \amalg \Lambda_{1}$ : there is not a smooth 1 -parameter family of legendrian links $\Theta_{t}, t \in[0,1]$, such that $\Theta_{0}=\Lambda_{1} \amalg \Lambda_{0}$ and $\Theta_{1}=\Lambda_{0} \amalg \Lambda_{1} . \Lambda_{0} \amalg \Lambda_{1}$ will be called the swap of $\Lambda_{1} \amalg \Lambda_{0}$.

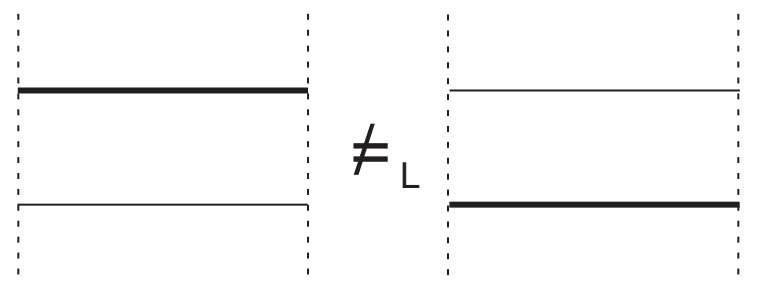

Figure 1.1: The legendrian link (0) and its nonequivalent swap

In this paper, more complicated legendrian links will be studied. All these links will be topologically unordered, but many will be legendrianly ordered. In addition, "flyping" moves applied to these links will produce topologically equivalent links that are legendrianly distinct. These more complicated links are constructed as the lifts of graphs of "multivalued" 1-periodic functions. Namely, in $\{(q, z): q \in I, z \in \mathbb{R}\}$, consider a piecewise smooth arc with semicubical cusps at the nonsmooth points. Then the arc has a well-defined tangent 
line at each point. As long as the tangent line is never vertical, and there are no self-tangencies, this arc will have a lift, with $p$ specified by the slope of the tangent line, to an embedded legendrian arc in $I \times \mathbb{R}^{2}$. As long as the appropriate boundary conditions are satisfied, this arc will lift to a legendrian knot in $\mathcal{J}^{1}\left(S^{1}\right)$. The graphs of such cusped curves can be seen in Figures 1.5, 1.12. Notice that it is not necessary to use broken curves to indicate which is the upperstrand in the lift: the third coordinate is determined by slope.

The basic links considered in this paper can be thought of as closures of rational tangles, defined by Conway in [6], and so it will be convenient to label the links using the rational tangle nomenclature developed in [20], which is similar to the nomenclature in [6] and [1]. With this notation, the ordered link $j^{1}(f) \amalg j^{1}(g)$ considered above will be called the link (0). More generally, for any $h \geq 0$, an "integral" link $(2 h)$ is constructed as the lift of the graphs of two functions $f, g: S^{1} \rightarrow \mathbb{R}$ that cross transversally at $2 h$ points. More complicated links will be described by length $2 n-1$ vectors of the form

$$
\left(2 h_{n}, v_{n-1}, \ldots, 2 h_{2}, v_{1}, 2 h_{1}\right), \quad h_{n}, v_{n-1}, \ldots, h_{2}, v_{1} \geq 1, \text { and } h_{1} \geq 0 .
$$

The standard rational legendrian link $\left(2 h_{n}, v_{n-1}, \ldots, 2 h_{2}, v_{1}, 2 h_{1}\right)$ can be constructed recursively: for $n=1$, these are the integral links $(2 h)$ described above, and for $n \geq 2$, the $(2 n-1)$-length link $\left(2 h_{n}, v_{n-1}, \ldots, 2 h_{2}, v_{1}, 2 h_{1}\right)$ is formed from "vertical and horizontal additions" to the $(2 n-3)$-length link $\left(2 h_{n}, \ldots, v_{2}, 2 h_{2}\right)$, as shown in Figure 1.3. This rational tangle nomenclature is extremely valuable in labeling knots and links in topological knot tables. Developing a legendrian version of such nomenclature is obviously useful for labeling legendrian knots and links in $\mathcal{J}^{1}\left(S^{1}\right)$, but also in $\mathbb{R}^{3}$ since, as described by $\mathrm{Ng}$ in [13], a satellite construction glues these links in $\mathcal{J}^{1}\left(S^{1}\right)$ into a tubular neighborhood of a knot in $\mathbb{R}^{3}$ to produce links in $\mathbb{R}^{3}$.

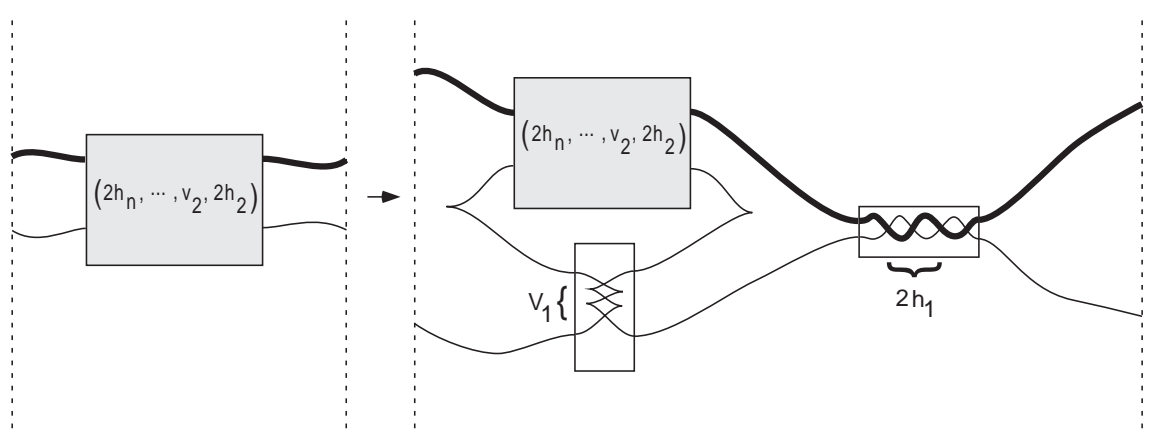

Figure 1.3: The recursive construction of the link $\left(2 h_{n}, v_{n-1}, \ldots, 2 h_{2}, v_{1}, 2 h_{1}\right)$ from the link $\left(2 h_{n}, v_{n-1}, \ldots, 2 h_{2}\right)$ 
When the link $\left(2 h_{n}, \ldots, v_{1}, 2 h_{1}\right)$ is thought of as a subset of $I \times \mathbb{R}^{2}$ without the boundary identification, the legendrian arcs topologically form a rational tangle that is alternatively known as the nonnegative rational number

(1.4) $q:=2 h_{1}+1 /\left(v_{1}+1 /\left(2 h_{2}+1 /\left(v_{2}+\cdots+1 /\left(v_{n-1}+1 / 2 h_{n}\right) \ldots\right)\right)\right) \in \mathbb{Q}$.

In fact, by Conway's construction, there is a topological tangle associated to every $q \in \mathbb{Q}$. Many of these tangles do not close up to two-component links in $\mathcal{J}^{1}\left(S^{1}\right)$. Other $q \in \mathbb{Q}$ will correspond to two-component topological links, but will not be considered here, since this paper will discuss only legendrian links where each component is legendrian isotopic to $j^{1}(0)$, the 1 -jet of the 0 -function. Such legendrian links will be called minimal legendrian links. It is shown in [20] that a minimal legendrian link version of $q \in \mathbb{Q}$ exists if and only if $q$ corresponds to a vector of the form in (1.2). Minimal legendrian links cannot be distinguished by examining the legendrian invariants for the strands known as the Thurston-Bennequin invariant and the rotation number. Background on these classic invariants can be found, for example, in [2], [7], [15].
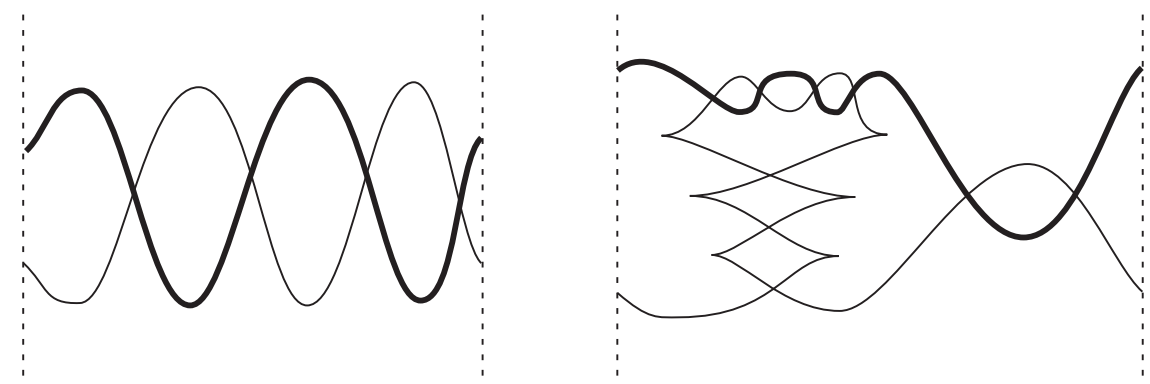

Figure 1.5: Minimal legendrian links 4 and $(4,3,2)=\frac{30}{13}$

For all the rational links, changing the order of the components produces topologically equivalent links. Although the legendrian link (0) is ordered, it is easy to verify that the legendrian link $(2 h)$, for $h \geq 1$, is not ordered. However, this is the exception among the rational legendrian links.

Theorem 1.6 (See Theorem 7.2) Consider the legendrian link $L=\left(2 h_{n}, v_{n-1}, 2 h_{n-1}, \ldots, v_{1}, 2 h_{1}\right)$. For $n=1, L=\left(2 h_{1}\right)$ is ordered iff $h_{1}=0$. For $n \geq 2, L$ is ordered for all choices of $h_{i}, v_{i}$.

In [19], the link (0) is shown to be ordered by studying Viterbo's invariants known as $c_{ \pm}$. These invariants arise as "canonical critical values" of a difference of generating functions associated to the strands of the link. The links of 
Theorem 1.6 with $h_{1}=0$ can also be distinguished by studying $c_{ \pm}$. All the links will be proven to be ordered by doing a more in depth analysis of the generating functions: rather than merely studying the critical values, homology groups $H_{k}^{ \pm}(L), k \in \mathbb{Z}$, for a minimal legendrian link $L$ will be constructed by examining the relative homology groups of canonical sublevel sets. This construction is explained in Section 3. From $H_{k}^{ \pm}[L]$, positive and negative homology polynomials are legendrian invariants associated to each link $L$ :

$$
\Gamma^{+}(\lambda)[L]=\sum_{k=-\infty}^{\infty} \operatorname{dim} H_{k}^{+}(L) \lambda^{k}, \quad \Gamma^{-}(\lambda)[L]=\sum_{k=-\infty}^{\infty} \operatorname{dim} H_{k}^{-}(L) \lambda^{k} .
$$

A comparison of $\Gamma^{+}(\lambda)[L]$ and $\Gamma^{-}(\lambda)[L]$ can detect that the legendrian link $L$ is ordered. It will be said that polynomials $\alpha(\lambda)=\sum_{k=-\infty}^{\infty} a_{k} \lambda^{k}$ and $\beta(\lambda)=$ $\sum_{k=-\infty}^{\infty} b_{k} \lambda^{k}$ are 1-shift palindromic if $\alpha(\lambda)=\lambda \cdot \overline{\beta(\lambda)}$, where $\overline{\beta(\lambda)}$ denotes the palindrome of $\beta: \overline{\beta(\lambda)}=\sum_{k=-\infty}^{\infty} b_{k} \lambda^{-k}$.

Theorem 1.8 (See Corollary 3.17) If $\Gamma^{+}(\lambda)[L]$ and $\Gamma^{-}(\lambda)[L]$ are not 1 -shift palindromic, then the link $L$ is ordered.

Theorem 1.6 then follows easily from Theorem 1.8 and the following calculation which is proven in Section 6 after developing algebraic topology tools in Section 4 and methods to calculate the indices of critical points in Section 5.

Theorem 1.9 (See Theorem 6.1) Consider the legendrian link $L=\left(2 h_{n}, v_{n-1}, \ldots, v_{1}, 2 h_{1}\right)$. Then

$$
\begin{aligned}
& \Gamma^{-}(\lambda)[L]=h_{1}+h_{2} \lambda^{-v_{1}}+h_{3} \lambda^{-v_{1}-v_{2}}+\cdots+h_{n} \lambda^{-v_{1}-v_{2}-\cdots-v_{n-1}}, \\
& \Gamma^{+}(\lambda)[L]= \begin{cases}\lambda \cdot \Gamma^{-}(\lambda)[L], & h_{1} \geq 1 \\
(1+\lambda)+\lambda \cdot \Gamma^{-}(\lambda)[L], & h_{1}=0 .\end{cases}
\end{aligned}
$$

In [14], the author and Lenny $\mathrm{Ng}$ find similar calculations of a refined version of the Chekanov first order polynomials; these Chekanov polynomials are invariants obtained from the differential algebras obtained from the theory of holomorphic curves, [5], [8], [10].

For the topological version of the link $\left(2 h_{n}, \ldots, v_{1}, 2 h_{1}\right), n \geq 2$, in addition to changing the order of the components, there are "flyping" moves that do not change the topological type of the link. A topological flype occurs when a portion of the link, represented by the circle labeled with " $F$ " in Figure 1.10, is rotated $180^{\circ}$ about a vertical axis (a vertical flype), or about a horizontal axis (a horizontal flype). For background on topological flypes, see, for example, [1]. 
This motivates the definition of a legendrian flype: when a crossing is formed by two edges emanating from a legendrian tangle, represented by the box labeled with "F" in Figure 1.10, a legendrian vertical (horizontal) flype occurs when the tangle is rotated $180^{\circ}$ about vertical (horizontal) axis and the crossing is "transferred" to the opposite edges. This rotation action is not a legendrian isotopy; so, although the resulting legendrian links are topologically equivalent, they are potentially not legendrianly equivalent.

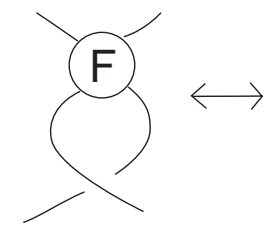

( a )

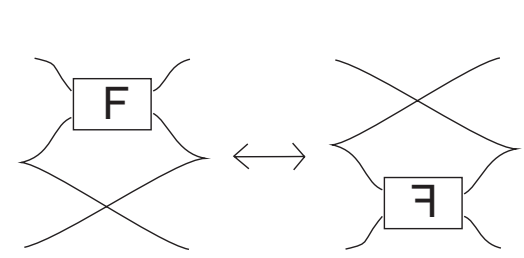

( c )

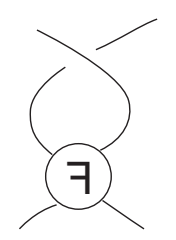

(a)

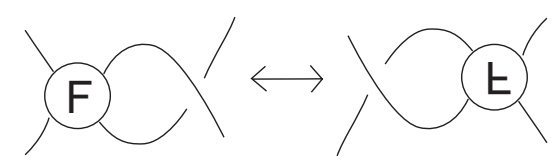

( b )

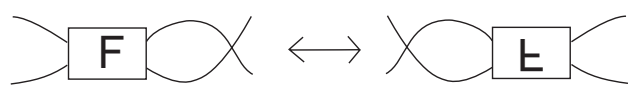

( d )

Figure 1.10: (a) a topological vertical flype, (b) a topological horizontal flype, (c) a legendrian vertical flype, (d) a legendrian horizontal flype.

For each positive horizontal entry $2 h_{i}, i \neq n$, in the legendrian link $\left(2 h_{n}, \ldots, 2 h_{2}, v_{1}, 2 h_{1}\right)$, it is possible to perform $0,1, \ldots$, or $2 h_{i}$ successive horizontal flypes; for each vertical entry $v_{i}$, it is possible to perform 0,1 , $\ldots$, or $v_{i}$ successive vertical flypes. A flype at the $2 h_{i}$ entry horizontally flips the tangle constructed from entries $2 h_{n}, \ldots, 2 h_{i+1}$, and $v_{i}$, while a flype at $v_{i}$ vertically flips the tangle made from entries $2 h_{n}, \ldots, 2 h_{i+1}$. The flyping procedure preserves the minimality of the links. The nomenclature

$$
\begin{aligned}
\left(2 h_{n}, v_{n-1}^{q_{n-1}}, 2 h_{n-1}^{p_{n-1}}, \ldots, 2 h_{2}^{p_{2}}, v_{1}^{q_{1}}, 2 h_{1}^{p_{1}}\right), & q_{i} \in\left\{0, \ldots, v_{i}\right\}, \\
& p_{i} \in\left\{0, \ldots, 2 h_{i}\right\},
\end{aligned}
$$

will be used to denote the modification of the standard link $\left(2 h_{n}, \ldots, 2 h_{2}, v_{1}\right.$, $2 h_{1}$ ) by $p_{i}$ horizontal flypes in the $i^{\text {th }}$ horizontal component, and $q_{i}$ vertical flypes in the $i^{\text {th }}$ vertical component. With this notation, the standard link $\left(2 h_{n}, \ldots, 2 h_{2}, v_{1}, 2 h_{1}\right)$ is written as $\left(2 h_{n}, v_{n-1}^{0}, 2 h_{n-1}^{0}, \ldots, 2 h_{2}^{0}, v_{1}^{0}, 2 h_{1}^{0}\right)$. If no superscript is specified for an entry of the vector, it will be assumed to be 0 . 
First consider a link $L$ that is obtained by applying vertical flypes to a standard rational link: $L=\left(2 h_{n}, v_{n-1}^{q_{n-1}}, 2 h_{n-1}, \ldots, v_{1}^{q_{1}}, 2 h_{1}\right), q_{i} \in\left\{0, \ldots, v_{i}\right\}$. Figure 1.12 illustrates some links that differ by vertical flypes. In fact, any link $L$ that is obtained from $L_{0}=\left(2 h_{n}, v_{n-1}, \ldots, v_{1}, 2 h_{1}\right)$ by vertical flypes is legendrianly equivalent to $L_{0}$.

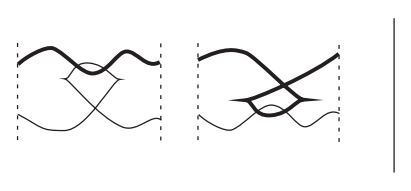

( a )
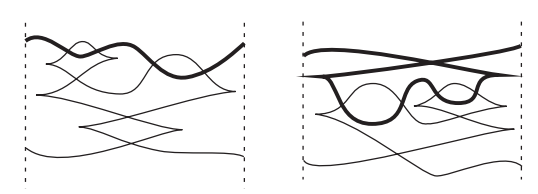

( b )

Figure 1.12: (a) The equivalent legendrian links $(2,1,0)$ and $\left(2,1^{1}, 0\right)$; (b) The equivalent legendrian links $(2,1,2,2,0),\left(2,1,2,2^{1}, 0\right)$, and $\left(2,1,2,2^{2}, 0\right)$.

Theorem 1.13 (See Theorem 2.1) Consider the legendrian links $L_{0}=\left(2 h_{n}, v_{n-1}, 2 h_{n-1}, \ldots, v_{1}, 2 h_{1}\right)$ and $L_{1}=\left(2 h_{n}, v_{n-1}^{q_{n-1}}, 2 h_{n-1}, \ldots, v_{1}^{q_{1}}, 2 h_{1}\right)$. Then $L_{0}$ and $L_{1}$ are legendrianly equivalent.

Theorem 1.13 is proved in Section 2 by showing that the $(q, z)$-projections of $L_{1}$ and $L_{0}$ are equivalent through a sequence of "legendrian planar isotopies" and "legendrian Reidemeister moves".

Next consider a link $L$ that is obtained by applying horizontal flypes to a standard rational link: $L=\left(2 h_{n}, v_{n-1}, 2 h_{n-1}^{p_{n-1}}, \ldots, v_{1}, 2 h_{1}^{q_{1}}\right), p_{i} \in\left\{0, \ldots, 2 h_{i}\right\}$. Figure 1.14 illustrates some links that differ by horizontal flypes. In contrast to the vertical flyping situation, it is possible to obtain distinct legendrian links by horizontal flypes. For example, the legendrian links $(2,1,2)$ and $\left(2,1,2^{1}\right)$ are topologically equivalent but not legendrianly equivalent. This is a consequence of calculating the $\Gamma^{-}(\lambda)$ or $\Gamma^{+}(\lambda)$ polynomials.

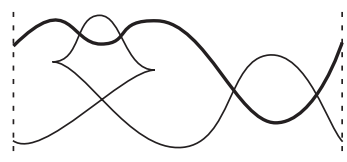

( a )

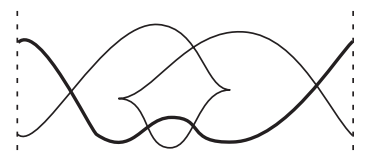

( b )

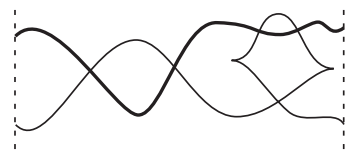

(c)

Figure 1.14: The legendrian links (a) $\left(2,1,2^{0}\right)$, (b) $\left(2,1,2^{1}\right)$, and (c) $\left(2,1,2^{2}\right)$. The link $\left(2,1,1^{0}\right)$ is equivalent to $\left(2,1,2^{2}\right)$, but distinct from $\left(2,1,2^{1}\right)$. 
Theorem 1.15 (See Theorem 6.2) Consider the legendrian link

$$
L=\left(2 h_{n}, v_{n-1}, 2 h_{n-1}^{p_{n-1}}, \ldots, v_{1}, 2 h_{1}^{p_{1}}\right), \quad p_{i} \in\left\{0, \ldots, 2 h_{i}\right\} .
$$

For $j=1, \ldots, n-1$, let $\sigma(j)=1+\sum_{i=1}^{j} p_{i} \bmod 2$. Then

$$
\begin{aligned}
& \Gamma^{-}(\lambda)[L]=h_{1}+\sum_{i=2}^{n} h_{i} \lambda^{(-1)^{\sigma(1)} v_{1}+(-1)^{\sigma(2)} v_{2}+\cdots+(-1)^{\sigma(i-1)} v_{i-1},} \\
& \Gamma^{+}(\lambda)[L]= \begin{cases}\lambda \cdot \Gamma^{-}(\lambda)[L], & h_{1} \geq 1 \\
(1+\lambda)+\lambda \cdot \Gamma^{-}(\lambda)[L], & h_{1}=0 .\end{cases}
\end{aligned}
$$

Remark/Question 1.16 Notice that given $L_{0}=\left(2 h_{n}, v_{n-1}, 2 h_{n-1}^{p_{n-1}}, \ldots, v_{1}\right.$, $\left.2 h_{1}^{p_{1}}\right)$ and $L_{1}=\left(2 h_{n}, v_{n-1}, 2 h_{n-1}^{w_{n-1}}, \ldots, v_{1}, 2 h_{1}^{w_{1}}\right)$, if $p_{i} \equiv w_{i} \bmod 2$, for all $i$, then $\Gamma^{-}(\lambda)\left[L_{0}\right]=\Gamma^{-}(\lambda)\left[L_{1}\right]$, and $\Gamma^{+}(\lambda)\left[L_{0}\right]=\Gamma^{+}(\lambda)\left[L_{1}\right]$. This is a natural condition on $p_{1}$ : if $w_{1} \equiv p_{1} \bmod 2$, and $w_{i}=p_{i}$ when $i \neq 1$, then $L_{0}$ and $L_{1}$ are, in fact, equivalent. Due to the boundary identification, "double" horizontal flypes are eqivalent to a rotation. It would be interesting to know if only the parity is important for other $p_{i}, i \neq 1$. For example, are the links $(2,1,2,1,0)$ and $\left(2,1,2^{2}, 1,0\right)$ illustrated in Figure 1.17 legendrianly equivalent?

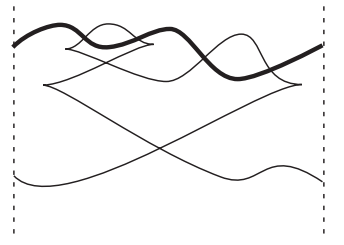

( a )

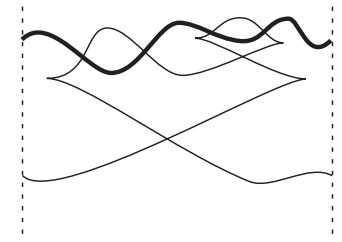

( b )

Figure 1.17: The legendrian links (a) $(2,1,2,1,0)$, and (b) $\left(2,1,2^{2}, 1,0\right)$. They have the same polynomials. Are they equivalent?

Remark/Question 1.18 In view of Theorem 1.6, it is natural to ask if, for $n \geq 2$, all the possible horizontal flypes of $L_{n}=\left(2 h_{n}, \ldots, v_{1}, 2 h_{1}\right)$ are also ordered. This is true when $n=2$ : for $n=2$, a flype is possible only when $h_{1} \geq 1$, and, in this case, it is easy to check that $L_{2}^{1}=\left(2 h_{2}, v_{1}, 2 h_{1}^{1}\right)$ is equivalent to the swap of $L_{2}^{0}=\left(2 h_{2}, v_{1}, 2 h_{1}^{0}\right)$. However, for $n \geq 3$, there are examples where the polynomials cannot detect if the link is ordered. For example, the link $L_{3}=\left(2,2,2^{1}, 1,2^{1}\right)$, illustrated in Figure 1.19, has $\Gamma^{-}(\lambda)\left[L_{3}\right]=\lambda^{-1}+$ $1+\lambda$, and, since $\Gamma^{-}(\lambda)$ is palindromic, Proposition 7.1 implies that $L_{3}$ is potentially unordered. Is $L_{3}$ equivalent to its swap? It is interesting to note that "slight modifications" of $L_{3}$ result in links that are ordered. For example, 
$L_{3}^{\prime}=\left(4,2,2^{1}, 1,2^{1}\right)$ and $L_{3}^{\prime \prime}=\left(2,1,2^{1}, 1,2^{1}\right)$ satisfy $\Gamma^{-}(\lambda)\left[L_{3}^{\prime}\right]=2 \lambda^{-1}+1+\lambda$, $\Gamma^{-}(\lambda)\left[L_{3}^{\prime}\right]=2+\lambda$, and, since these polynomials are not palindromic, $L_{3}^{\prime}$ and $L_{3}^{\prime \prime}$ are each ordered.
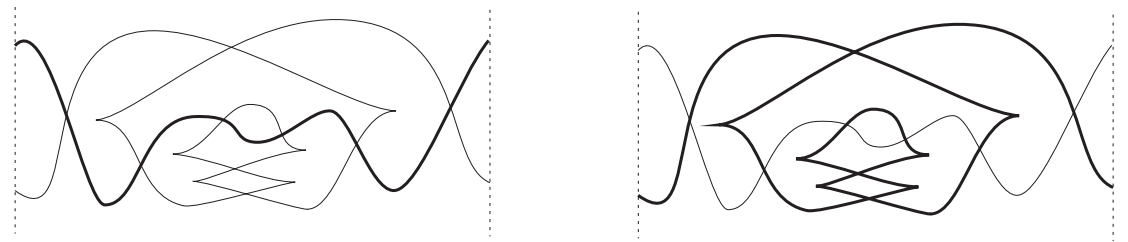

Figure 1.19: The link $\left(2,2,2^{1}, 1,2^{1}\right)$ and its swap $\overline{\left(2,2,2^{1}, 1,2^{1}\right)}$. Their polynomials are the same. Are the links equivalent?

Remark/Question 1.20 See Corollary 2.3 and Proposition 7.3 The flyping procedure can be thought of as a generalization of the swapping procedure: as shown in Corollary 2.3, for $h_{1} \geq 1$, if

$$
\begin{gathered}
L_{0}=\left(2 h_{n}, v_{n-1}, \ldots, 2 h_{2}, v_{1}, 2 h_{1}\right) \text { and } L_{1}=\left(2 h_{n}, v_{n-1}, \ldots, 2 h_{2}, v_{1}, 2 h_{1}^{1}\right), \\
\text { where } v_{1}, \ldots, v_{n-2} \equiv 0 \bmod 2,
\end{gathered}
$$

then $L_{0}$ and $L_{1}$ are swaps of one another. This motivates two questions. Is this statement true without the hypothesis on the parity of $v_{i}$ ?; in particular, is $\left(2,1,2,1,2^{1}\right)$ equivalent to the swap of $(2,1,2,1,2)$ ? In fact, $\left(2,1,2,1,2^{1}\right)$ is equivalent to the swap of $\left(2,1,2^{2}, 1,2\right)$, and thus this question is closely related to Remark/Question 1.16. Secondly, Is the analog of this statement true for horizontal flypes of $L_{0}$ ? Namely, for $h_{1} \geq 1$, consider

$$
\begin{aligned}
& M_{0}=\left(2 h_{n}, v_{n-1}, 2 h_{n-1}^{p_{n-1}}, \ldots, 2 h_{2}^{p_{2}}, v_{1}, 2 h_{1}^{p_{1}}\right), \\
& M_{1}=\left(2 h_{n}, v_{n-1}, 2 h_{n-1}^{p_{n-1}}, \ldots, 2 h_{2}^{p_{2}}, v_{1}, 2 h_{1}^{p_{1}+1}\right) .
\end{aligned}
$$

Let $\overline{M_{0}}$ be the swap of $M_{0}$. It is shown in Proposition 7.3 that $\Gamma^{-}(\lambda)\left[\overline{M_{0}}\right]=$ $\Gamma^{-}(\lambda)\left[M_{1}\right]$, and $\Gamma^{+}(\lambda)\left[\overline{M_{0}}\right]=\Gamma^{+}(\lambda)\left[M_{1}\right]$. Are $\overline{M_{0}}$ and $M_{1}$ equivalent? In particular, are $\overline{\left(2,2,2^{1}, 2,2\right)}$ and $\left(2,2,2^{1}, 2,2^{1}\right)$ equivalent? See Figure 1.21.

Remark/Question 1.22 The question of the equivalence of the links $\overline{\left(2,2,2^{1}, 2,2\right)}$ and $\left(2,2,2^{1}, 2,2^{1}\right)$ mentioned in the previous remark is closely related to the question of the equivalence of the links $L_{0}=\left(2,1,2^{1}, 1,0\right)$ and $L_{1}=\left(2,1,2^{1}, 1^{1}, 0\right)$; see Figure 1.23. Notice that $L_{1}$ only differs from $L_{0}$ by a vertical flype, but $L_{0}$ is not standard, and thus Theorem 1.13 does not imply they are equivalent. Remark 6.3 explains that the $\Gamma^{+}(\lambda)$ and $\Gamma^{-}(\lambda)$ polynomials will never be able to distinguish two links that only differ by vertical 


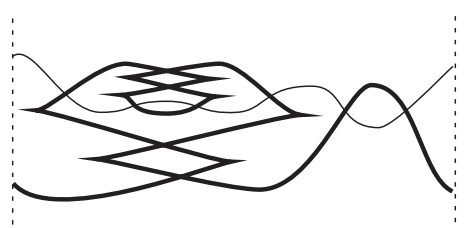

( a )

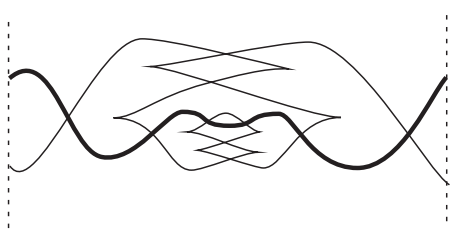

( b )

Figure 1.21: The legendrian links (a) $\overline{\left(2,2,2^{1}, 2,2\right)}$, (b) $\left(2,2,2^{1}, 2,2^{1}\right)$. These links have the same polynomials. Are they equivalent?

flypes. It would be interesting to know if it is ever possible to obtain distinct legendrian links by a vertical flype: Do there exist links of the form $L_{0}=$ $\left(2 h_{n}, v_{n-1}^{q_{n-1}}, 2 h_{n-1}^{p_{n-1}}, \ldots, v_{1}^{q_{1}}, 2 h_{1}^{p_{1}}\right)$ and $L_{1}=\left(2 h_{n}, v_{n-1}^{w_{n-1}}, 2 h_{n-1}^{p_{n-1}}, \ldots, v_{1}^{w_{1}}, 2 h_{1}^{p_{1}}\right)$ such that $L_{0}$ and $L_{1}$ are not legendrianly equivalent? Lemma 2.1 .1 implies that if $w_{i}=q_{i}$, when $i \neq n-1$, then $L_{0}$ and $L_{1}$ are equivalent.

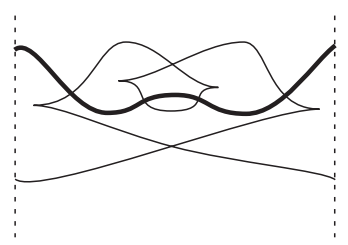

( a )

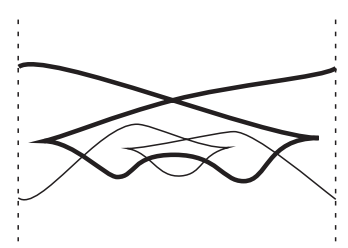

( b )

Figure 1.23: The links (a) $\left(2,1,2^{1}, 1,0\right)$, and (b) $\left(2,1,2^{1}, 1^{1}, 0\right)$. They have the same polynomials. Are they equivalent?

The following summarizes how many different legendrian representations of a given rational link type can be constructed from the swap and the flype operations. It would be interesting to know if there are other minimal legendrian versions of these links.

Theorem 1.24 (See Theorems 7.2, 7.4, 7.6) Consider the topological link $L_{n}=\left(2 h_{n}, v_{n-1}, 2 h_{n-1}, \ldots, 2 h_{2}, v_{1}, 2 h_{1}\right), \quad h_{n}, v_{n-1}, \ldots, h_{2}, v_{1} \geq 1, \quad h_{1} \geq 0$.

(1) If $n=2$, there are at least 2 legendrianly distinct minimal links that are topologically equivalent to $L_{2}$.

(2) If $n=3$, and either $h_{1}=0, h_{2} \neq h_{3}$, or $v_{2} \neq 2 v_{1}$, then there are at least 4 legendrianly distinct minimal links that are topologically equivalent to $L_{3}$. 
(3) For $n \geq 4$, if $\max \left\{h_{1}, 1\right\} \cup\left\{h_{i}\right\}_{i=2}^{n}$ form a set of order $n$ such that the sums of all its $2^{n}$ subsets are distinct, there are at least $2^{n-1}$ minimal legendrian links that are topologically equivalent to $L_{n}$.

When $h_{1} \geq 1$, these $2^{n-1}$ different legendrian links arise by looking at

$$
\left(2 h_{n}, v_{n-1}, 2 h_{n-1}^{p_{n-1}}, \ldots, 2 h_{2}^{p_{2}}, v_{1}, 2 h_{1}^{p_{1}}\right)
$$

for $p_{i} \in\{0,1\}, i=1, \ldots, n-1$. When $h_{1}=0$, the variations are obtained by the original and the swap of each of the $2^{n-2}$ links in (1.25).

Remark/Question 1.26 The condition that $h_{1}=0, h_{2} \neq h_{3}$, or $v_{2} \neq 2 v_{1}$ when $n=3$, or that, for $n \geq 4,\left\{h_{i}\right\}$ or $\left\{h_{i}\right\} \cup\{1\}$ form a set of order $n$ with "distinct subset sums" guarantees that distinct polynomials are associated to the $2^{n-1}$ links in (1.25). In contrast, consider

$$
L^{(0,1,0)}=\left(2,1,2^{0}, 1,2^{1}, 1,2^{0}\right), \quad L^{(0,1,1)}=\left(2,1,2^{0}, 1,2^{1}, 1,2^{1}\right) ;
$$

see Figure 1.27. By Theorem 1.15,

$$
\Gamma^{-}(\lambda)\left[L^{(0,1,0)}\right]=\lambda^{-1}+2+\lambda=\Gamma^{-}(\lambda)\left[L^{(0,1,1)}\right] .
$$

Are $L^{(0,1,0)}$ and $L^{(0,1,1)}$ legendrianly equivalent? It is interesting to note that variations of $L^{(0,1,0)}$ and $L^{(0,1,1)}$ with $h_{2} \neq h_{4}$ will produce different polynomials. For example, if

$$
M^{(0,1,0)}=\left(2,1,2^{0}, 1,4^{1}, 1,2^{0}\right), \quad M^{(0,1,1)}=\left(2,1,2^{0}, 1,4^{1}, 1,2^{1}\right),
$$

then $\Gamma^{-}(\lambda)\left[M^{(0,1,0)}\right]=2 \lambda^{-1}+2+\lambda$, while $\Gamma^{-}(\lambda)\left[M^{(0,1,1)}\right]=\lambda^{-1}+2+2 \lambda$.
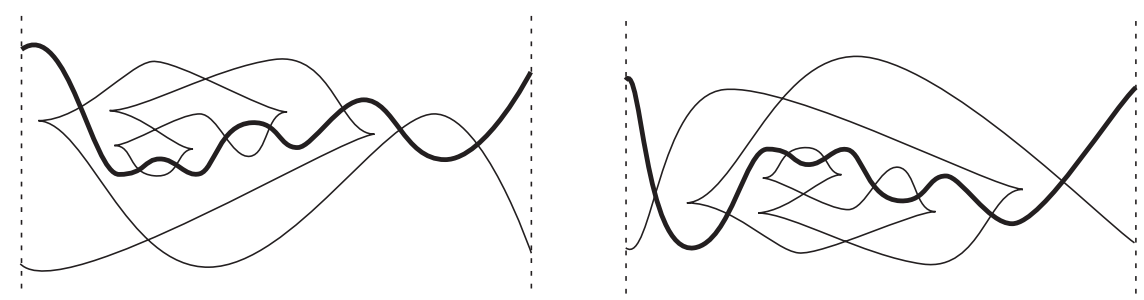

Figure 1.27: The legendrian links $L^{(0,1,0)}=\left(2,1,2^{0}, 1,2^{1}, 1,2^{0}\right)$ and $L^{(0,1,1)}=$ $\left(2,1,2^{0}, 1,2^{1}, 1,2^{1}\right)$. They have the same polynomials. Are they equivalent? 
Remark 1.28 For certain choices of $v_{i}$, it is possible that the $2^{n-1}$ links $L_{n}=\left(2 h_{n}, v_{n-1}, 2 h_{n-1}^{p_{n-1}}, \ldots, v_{2}, 2 h_{1}^{p_{1}}\right)$ have distinct polynomials without the hypothesis that $\left\{h_{1}, \ldots, h_{n}\right\}$ form a set of order $n$ with distinct subset sums. For example, consider $L_{4}=(2,3,2,2,2,1,2)$. In this example, each flype gives a polynomial containing a different set of powers of $t$. More generally, given $v_{1}, \ldots, v_{n}$, if the $2^{n-1}$ sets

$$
\begin{aligned}
& \left\{(-1)^{\sigma(1)} v_{1},(-1)^{\sigma(1)} v_{1}+(-1)^{\sigma(2)} v_{2}, \ldots,\right. \\
& \left.\quad(-1)^{\sigma(1)} v_{1}+(-1)^{\sigma(2)} v_{2}+\cdots+(-1)^{\sigma(n-1))} v_{n-1}\right\}, \quad \sigma:\{1, \ldots, n-1\} \rightarrow \mathbb{Z}_{2}
\end{aligned}
$$

are distinct, then any choice of $h_{i}$ will produce $2^{n-1}$ different $\Gamma^{-}(\lambda)$ polynomials.

In Section 6, the polynomials are calculated for rational links, their flypes, and for the usually nonrational "connect sums" of such links. Since, up to legendrian isotopy, the connect sum may depend on the choice of where the links are cut into tangles, a standard position for cutting the links will be chosen. Namely, the connect sum $L_{1} \# L_{2}$ is defined as the closure of the connect sum of the legendrian rational tangles : $L_{1}$ : and : $L_{2}$ :, which are constructed analogously to the links $L_{i}$. This construction is illustrated in Figure 1.29 where, if $L_{1}$ denotes the link $\left(2 h_{n}, \ldots, 2 h_{1}\right)$, then $: L_{1}$ : corresponds to Figure 1.3 except considered as a tangle rather than closed to a link.
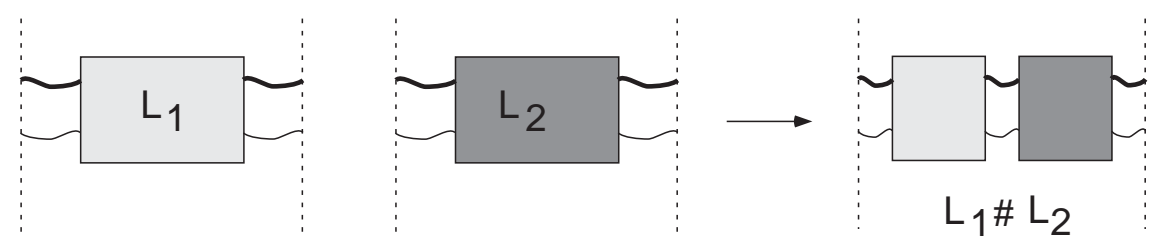

Figure 1.29: The construction of the connect sum $L_{1} \# L_{2}$

Theorem 1.30 (See Theorem 6.4) Consider the legendrian links

$$
\begin{gathered}
L_{1}=\left(2 h_{n}, v_{n-1}, 2 h_{n-1}^{p_{n-1}}, \ldots, v_{1}, 2 h_{1}^{p_{1}}\right), \\
L_{2}=\left(2 k_{m}, u_{m-1}, 2 k_{m-1}^{w_{m-1}}, \ldots, u_{1}, 2 k_{1}^{w_{1}}\right) .
\end{gathered}
$$

Then

$$
\begin{array}{ll}
\Gamma^{-}(\lambda)\left[L_{1} \# L_{2}\right]=\Gamma^{-}(\lambda)\left[L_{1}\right]+\Gamma^{-}(\lambda)\left[L_{2}\right] \\
\Gamma^{+}(\lambda)\left[L_{1} \# L_{2}\right]= \begin{cases}\Gamma^{+}(\lambda)\left[L_{1}\right]+\Gamma^{+}(\lambda)\left[L_{2}\right], & h_{1}, k_{1} \geq 1 \\
\Gamma^{+}(\lambda)\left[L_{1}\right]+\Gamma^{+}(\lambda)\left[L_{2}\right]-(1+\lambda), & \text { else. }\end{cases}
\end{array}
$$


Remark/Question 1.31 Theorem 1.30 gives many examples of topogically equivalent, nonrational minimal links that are legendrianly distinct; it also raises some interesting questions. For example, Are the legendrian links $(2,1,2) \#(2,1,2)$ and $\left(2,1,2^{2}\right) \#(2,1,2)$ equivalent? See Figure 1.32. Notice that the rotation that made $(2,1,2)$ and $\left(2,1,2^{2}\right)$ equivalent is no longer possible.
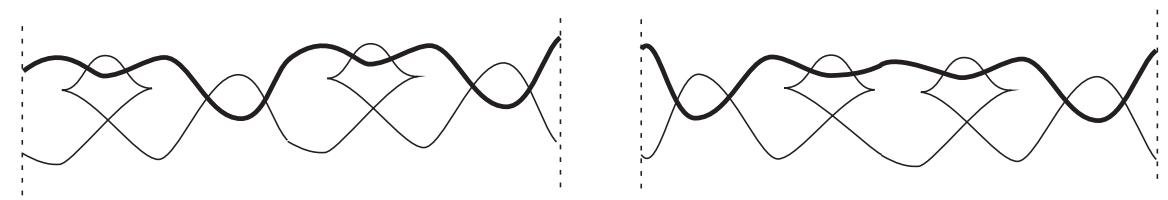

Figure 1.32: The legendrian links $(2,1,2) \#(2,1,2)$, and $\left(2,1,2^{2}\right) \#(2,1,2)$. They have the same polynomials. Are they equivalent?

Lastly, notice that the nomenclature for links in $\mathcal{J}^{1}\left(S^{1}\right)$ easily lends itself to nomenclature for legendrian knots in $\mathcal{J}^{1}\left(S^{1}\right)$. In analog with (1.2), length $2 n-1$ vectors of the form

$$
\begin{aligned}
\left(2 h_{n}, v_{n-1}^{q_{n-1}}, 2 h_{n-1}^{p_{n-1}}, \ldots, 2 h_{2}^{p_{2}}, v_{1}^{q_{1}}, 2 h_{1}^{p_{1}}-1\right), & \\
h_{n}, v_{n-1}, \ldots, h_{2}, v_{1}, h_{1} \geq 1, & q_{i} \in\left\{0, \ldots, v_{i}\right\}, \\
& p_{i} \in\left\{0, \ldots, 2 h_{i}\right\},
\end{aligned}
$$

give rise to legendrian knots. The technique of generating functions, as used in this paper, no longer applies. The above results about links raise many interesting questions about such knots. For example, Are the legendrian knots $(2,1,2,1,1)$ and $\left(2,1,2^{1}, 1,1\right)$ legendrianly equivalent? See Figure 1.34.
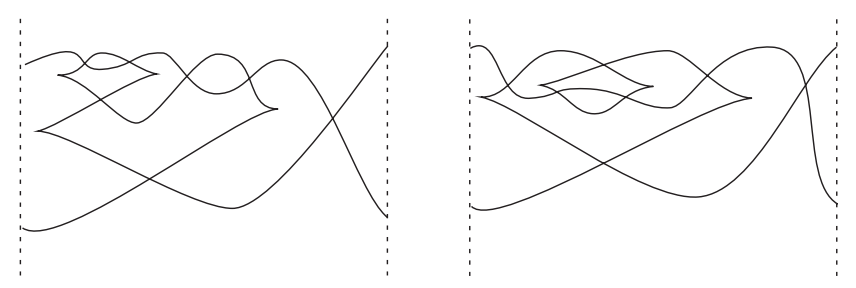

Figure 1.34: The legendrian knots $(2,1,2,1,1)$ and $\left(2,1,2^{1}, 1,1\right)$. They have the same classical invariants. Are they equivalent?

In [14], it is shown that sometimes the differential algebra approach can say something about some of the above questions. It is a topic for further study 
to understand if the generating function approach can be further refined to capture as many invariants as the holomorphic curve approach.

\section{Equivalent Vertical Flypes}

In this section, it is shown that it is not possible to produce a nonequivalent legendrian link by performing vertical flypes to a standard link. Recall the terminology $\left(2 h_{n}, v_{n-1}^{q_{n-1}}, 2 h_{n-1}, \ldots, v_{1}^{q_{1}}, 2 h_{1}\right), q_{i} \in\left\{0, \ldots, v_{i}\right\}$, introduced in (1.11).

Theorem 2.1 Consider the legendrian links $L_{0}=\left(2 h_{n}, v_{n-1}, 2 h_{n-1}, \ldots, v_{1}\right.$, $\left.2 h_{1}\right)$ and $L_{1}=\left(2 h_{n}, v_{n-1}^{q_{n-1}}, 2 h_{n-1}, \ldots, v_{1}^{q_{1}}, 2 h_{1}\right)$. Then $L_{0}$ and $L_{1}$ are legendrianly equivalent.

To prove Theorem 2.1, it suffices to show that the $(q, z)$-projections of the links are equivalent by a sequence of "legendrian planar isotopies" and "legendrian (Reidemeister) moves"; see Figure 2.2. (For background on the topological Reidemeister moves, see, for example, [1].) A legendrian planar isotopy is a planar isotopy that does not introduce cusps or vertical tangents. Each of the legendrian type $1^{ \pm}$moves are analogous to one of the type I topological Reidemeister moves: one additional crossing and two additional cusps are introduced into the projection. The legendrian type 2 moves are analogous to the type II Reidemeister moves: two new crossings are introduced into the projection after a cusp crosses a noncusped segment. Note that the relative slopes of the cusp and the segment determine if the cusped segment passes over or under the noncusped segment. Lastly, the legendrian type 3 move is analogous to one of the type III Reidemeister moves: a strand is slid from one side of a crossing to the other.

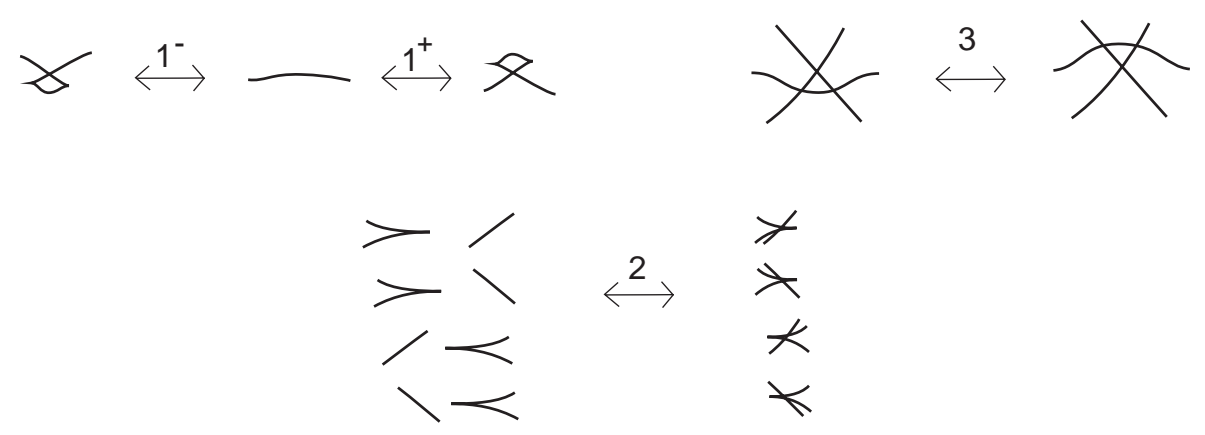

Figure 2.2: The legendrian Reidemeister moves 
For the proof of Theorem 2.1, it will be useful to introduce the notion of a legendrian tangle, [20]. A legendrian tangle consists of two disjoint legendrian $\operatorname{arcs} \Lambda_{1}, \Lambda_{0} \subset \mathcal{J}^{1}([0,1])$, where $\mathcal{J}^{1}([0,1])$ denotes the 1 -jet space of the interval $[0,1]$, with $\partial \Lambda_{1}, \partial \Lambda_{0} \subset\{q=0\} \cup\{q=1\}$. Legendrian tangles $T_{1}, T_{2}$ are equivalent if their $(q, z)$-projections are equivalent by a sequence of legendrian planar isotopies and legendrian moves supported in $(0,1) \times \mathbb{R}$. The nomenclature : $2 h_{n}, v_{n-1}, \ldots, 2 h_{1}$ : will be used to denote the legendrian tangle constructed using the same recursive procedure used to construct the legendrian link $\left(2 h_{n}, v_{n-1}, \ldots, 2 h_{1}\right)$.

Proof of Theorem 2.1 For $n \geq 2$, the desired equivalence of the links $\left(2 h_{n}, v_{n-1}, \ldots, v_{1}, 2 h_{1}\right)$ and $\left(2 h_{n}, v_{n-1}^{q_{n-1}}, \ldots, v_{1}^{q_{1}}, 2 h_{1}\right)$ will follow from a proof that the tangles : $2 h_{n}, v_{n-1}, \ldots, v_{1}, 2 h_{1}:$ and $: 2 h_{n}, v_{n-1}^{q_{n-1}}, \ldots, v_{1}^{q_{1}}, 2 h_{1}:$ are equivalent for all choices of $q_{i} \in\left\{0, \ldots, v_{i}\right\}, i=1, \ldots, n-1$. The equivalence of the tangles will be proved by induction on $n$. Lemma 2.1.1 proves the base case of $n=2$.

Lemma 2.1.1 The legendrian tangles : $2 h_{2}, v_{1}, 2 h_{1}:$ and $: 2 h_{2}, v_{1}^{q_{1}}, 2 h_{1}:$ are equivalent for any $q_{1} \in\left\{0, \ldots, v_{1}\right\}$.

Proof It suffices to show that the tangles : $2 h, 1,0:$ and $: 2 h, 1^{1}, 0:$ are equivalent for all $h \geq 1$. This will be shown by an induction argument on $h$. Figure 2.1.1.1 outlines the legendrian moves that prove the base case of $h=1$.

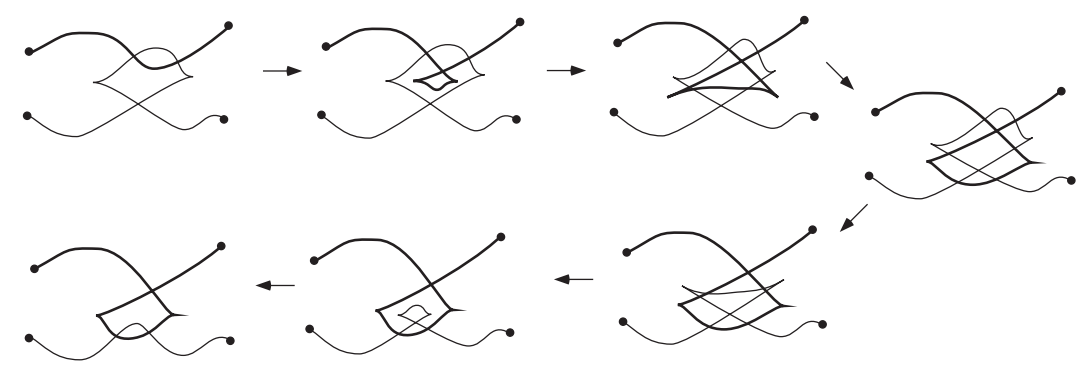

Figure 2.1.1.1: The equivalence of the tangles : $2,1,0:$ and $: 2,1^{1}, 0$ :

As the induction step, assume $: 2 k, 1,0:$ and $: 2 k, 1^{1}, 0:$ are equivalent. Figure 2.1.1.2 then outlines the legendrian moves that demonstrate the equivalence of $: 2 k+2,1,0:$ and $: 2 k+2,1^{1}, 0:$.

This completes the proof of Lemma 2.1.1. 


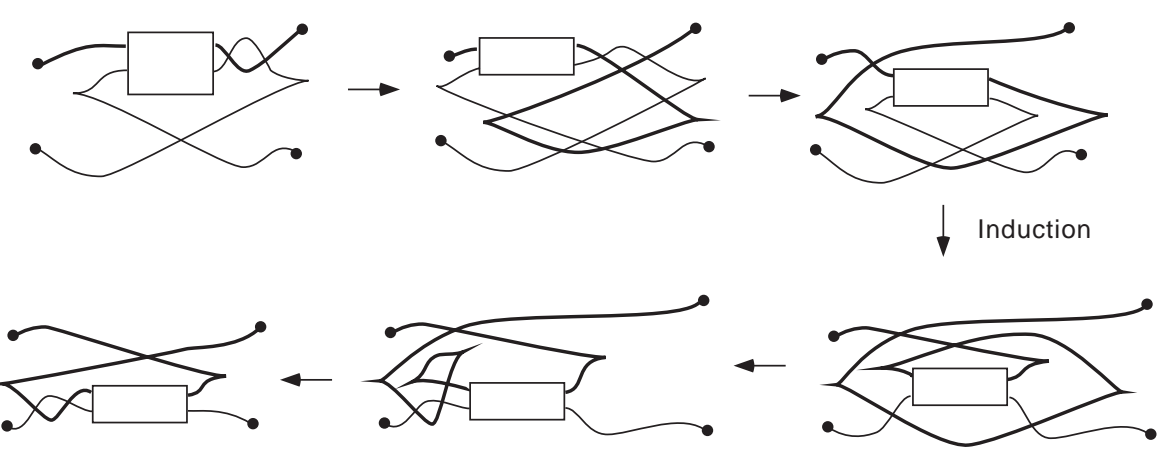

Figure 2.1.1.2: The equivalence of the tangles : $2 k+2,1,0:$ and $: 2 k+2,1^{1}, 0$ : assuming the equivalence of : $2 k, 1,0:$ and $: 2 k, 1^{1}, 0$ :

The induction step in the proof of Theorem 2.1 is to show that the length $2 n-1$ tangles : $2 h_{n}, v_{n-1}, \ldots, v_{2}, 2 h_{2}, v_{1}, 2 h_{1}:$ and $: 2 h_{n}, v_{n-1}^{q_{n-1}}, \ldots, v_{2}^{q_{2}}, 2 h_{2}, v_{1}^{q_{1}}, 2 h_{1}$ : are equivalent for all choices of $q_{i}, i=1, \ldots, n-1$, assuming that the length $2 n-3$ tangles : $2 h_{n}, v_{n-1}, \ldots, v_{2}, 2 h_{2}:$ and $: 2 h_{n}, v_{n-1}^{q_{n-1}}, \ldots, v_{2}^{q_{2}}, 2 h_{2}:$ are equivalent for all choices of $q_{i}, i=2, \ldots, n-1$. For this, it suffices to prove that $: 2 h_{n}, v_{n-1}, \ldots, v_{2}, 2 h_{2}, 1,0:$ and $: 2 h_{n}, v_{n-1}, \ldots, v_{2}, 2 h_{2}, 1^{1}, 0:$ are equivalent. This will be proved by induction on $h_{2}$. Figure 2.1.2 outlines the moves that prove the base case statement that : $2 h_{n}, v_{n-1}, \ldots, v_{2}, 2,1,0:$ and $: 2 h_{n}, v_{n-1}, \ldots, v_{2}, 2,1^{1}, 0:$ are equivalent when $v_{2} \geq 2$. The figure is easily modified to prove the case $v_{2}=1$.

The induction statement is that the equivalence of the tangles

$: 2 h_{n}, v_{n-1}, \ldots, v_{2}, 2 k+2,1,0:$ and $: 2 h_{n}, v_{n-1}, \ldots, v_{2}, 2 k+2,1^{1}, 0:$ follows from the equivalence of the tangles : $2 h_{n}, v_{n-1}, \ldots, v_{2}, 2 k, 1,0:$ and $: 2 h_{n}, v_{n-1}^{q_{n-1}}, \ldots, v_{2}^{q_{2}}, 2 k, 1^{1}, 0$ : This is proven using a sequence of moves similar to those shown in Figure 2.1.1.2.

A nice consequence of Theorem 2.1 is that the flyping procedure can be seen as a generalization of the swapping procedure.

Corollary 2.3 For $h_{1} \geq 1$, consider the legendrian links

$$
L_{0}=\left(2 h_{n}, v_{n-1}, \ldots, 2 h_{2}, v_{1}, 2 h_{1}\right), \quad L_{1}=\left(2 h_{n}, v_{n-1}, \ldots, 2 h_{2}, v_{1}, 2 h_{1}^{1}\right),
$$

when $v_{1}, \ldots, v_{n-2}$ are even. If $\overline{L_{0}}$ denotes the swap of $L_{0}$, then $\overline{L_{0}}$ and $L_{1}$ are equivalent legendrian links. 


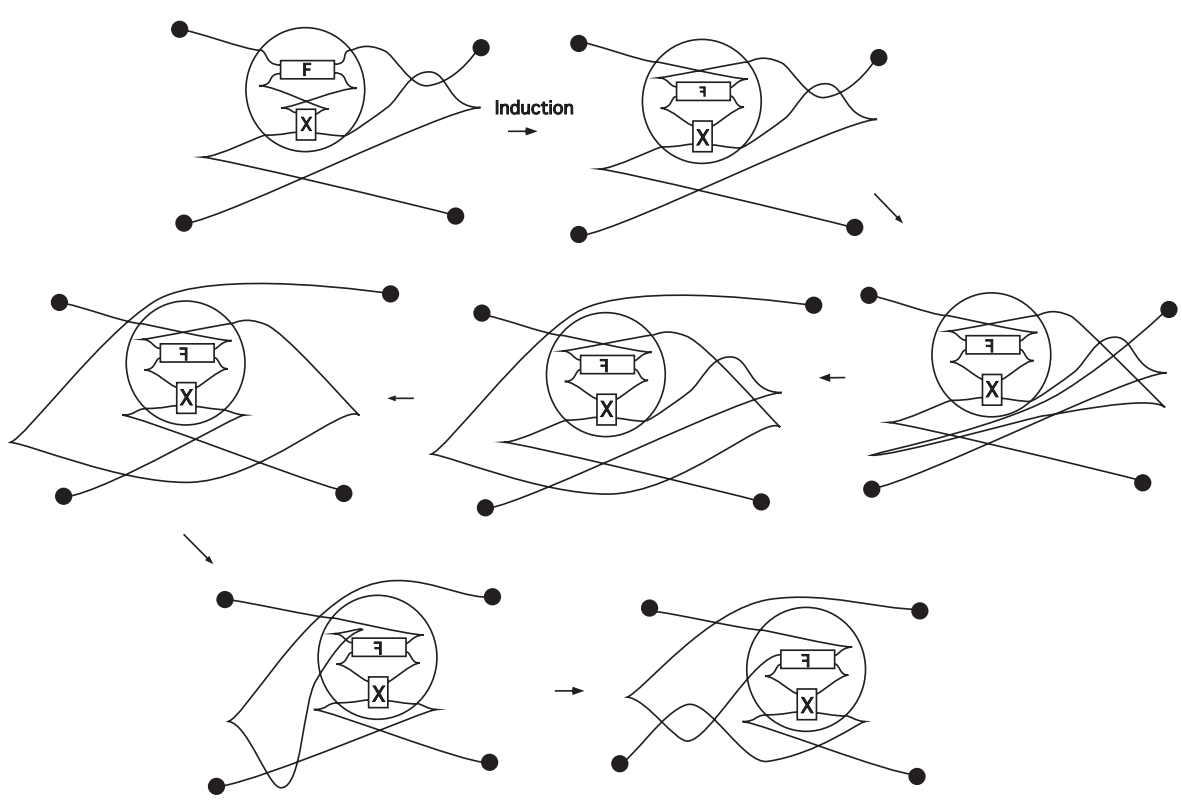

Figure 2.1.2: The equivalence of the tangles : $2 h_{n}, v_{n-1}, \ldots, v_{2}, 2,1,0:$ and : $2 h_{n}, v_{n-1}, \ldots, v_{2}, 2,1^{1}, 0$ : assuming the equivalence of the tangles $: 2 h_{n}, v_{n-1}, \ldots, v_{2}, 0:$ and $: 2 h_{n}, v_{n-1}, \ldots, v_{2}^{q_{2}}, 0$ :

Proof By a rotation, $L_{1}$ is equivalent to the swap of

$$
\left(2 h_{n}, v_{n-1}^{v_{n-1}}, 2 h_{n-1}^{p_{n-1}}, v_{n-2}^{v_{n-2}}, \ldots, 2 h_{2}^{p_{2}}, v_{1}^{v_{1}}, 2 h_{1}\right),
$$

where, for $i=2, \ldots, n-1$,

$$
p_{i}=\left\{\begin{array}{ll}
0, & \tau(i) \equiv 0 \bmod 2 \\
2 h_{i}, & \tau(i) \equiv 1 \bmod 2
\end{array}, \quad \text { where } \tau(i)=\sum_{k=1}^{i-1} v_{k} .\right.
$$

Thus, if $v_{1}, \ldots, v_{n-2}$ are even, $L_{1}$ is equivalent to the swap of $\left(2 h_{n}, v_{n-1}^{v_{n-1}}, 2 h_{n-1}, v_{n-2}^{v_{n-2}}, \ldots, 2 h_{2}, v_{2}^{v_{2}}, 2 h_{1}\right)$, and thus, by Theorem $2.1, L_{1}$ is equivalent to the swap of $L_{0}$.

\section{Generating Function Theory}

Recall that the links in $\mathcal{J}^{1}\left(S^{1}\right)$ under consideration are minimal, and thus, by definition, each strand is legendrian isotopic to $j^{1}(0)$, the 1 -jet of the 0 function. This condition guarantees that each component of the link has an essentially unique "generating function". The technique of generating functions 
is an extension of the fact that the 1-jet of a smooth function, $f:\left\{q \in S^{1}\right\} \rightarrow$ $\{z \in \mathbb{R}\}$, is a legendrian submanifold. More generally, if $F: S^{1} \times \mathbb{R}^{N} \rightarrow \mathbb{R}$ has fiber derivatives $\frac{\partial F}{\partial e}$ transverse to 0 , then

$$
\Lambda:=\left\{\left(q_{0}, \frac{\partial F}{\partial q}\left(q_{0}, e_{0}\right), F\left(q_{0}, e_{0}\right)\right): \frac{\partial F}{\partial e}\left(q_{0}, e_{0}\right)=0\right\},
$$

is an immersed legendrian submanifold of $\mathcal{J}^{1}\left(S^{1}\right)$, and $F$ is called a generating function for $\Lambda$. Critical points of a generating function correspond to points where $\Lambda$ intersects $\{p=0\}$. A function $F: S^{1} \times \mathbb{R}^{N} \rightarrow \mathbb{R}$ is said to be quadratic at infinity if there exists a fiberwise quadratic, nondegenerate form $Q(q, e)$ such that $F(q, e)-Q(q, e)$ has compact support. The index of a quadratic at infinity function will refer to the index of the associated quadratic form. The abbreviation g.q.i. function will be used to denote a generating and quadratic at infinity function. There is a parallel definition of quadratic at infinity generating functions for lagrangian submanifolds of cotangent bundles; see, for example, [21], [16], [22], [18], [9].

The following existence theorem is proved, for a more general situation, by Chaperon in [3], by Chekanov in [4], and in the appendix to [19].

Existence (3.2) If $\Lambda_{t} \subset \mathcal{J}^{1}\left(S^{1}\right), t \in[0,1]$, is a smooth 1-parameter family of legendrian submanifolds such that $\Lambda_{0}=j^{1}(0)$, then there exists an $N \in \mathbb{N}$, and a smooth 1-parameter family $F_{t}: S^{1} \times \mathbb{R}^{N} \rightarrow \mathbb{R}, t \in[0,1]$, of g.q.i. functions for $\Lambda_{t}$.

Example 3.3 For the strands of the links under consideration in this paper, generating functions can be explicitly described. For example, if the $(q, z)-$ projection of $\Lambda, \pi_{q, z}(\Lambda)$, is the graph of a function $f$, then $f: S^{1} \rightarrow \mathbb{R}$ is a g.q.i. function for $\Lambda$. Notice that if $Q(e)$ is a quadratic form, $Q(e)=\sum \alpha_{i j} e_{i} e_{j}$, then $F(q, e)=f(q)+Q(e)$ is also a g.q.i. function for $\Lambda$. Next consider $\Lambda$ given as the "nongraph" strand the link $\left(2,1^{1}, 0\right)$ as pictured in Figure 1.12. Construct a g.q.i. function $F: S^{1} \times \mathbb{R} \rightarrow \mathbb{R}$ for this strand with a "bubble" as follows. On $\{q=0\}$, let the fiber function $F(0, \cdot): \mathbb{R} \rightarrow \mathbb{R}$ be a quadratic function of index 1 with critical point $a$ with value given by $\pi_{q, z}(\Lambda) \cap\{q=0\}$. For $q_{0}$ in a neighborhood of $0, F\left(q_{0}, \cdot\right): \mathbb{R} \rightarrow \mathbb{R}$ continues to be a quadratic function, and there is a path of critical points $a\left(q_{0}\right) \in\left\{q_{0}\right\} \times \mathbb{R}$ of $F\left(q_{0}, \cdot\right)$ with values given by $\pi_{q, z}(\Lambda) \cap\left\{q=q_{0}\right\}$. At the $q$-coordinate where the left cusp occurs, the fiber function experiences a birth of a degenerate critical point. This can be accomplished by a compact perturbation of the function. As $q$ increases, this degenerate critical point bifurcates into two paths of nondegenerate critical points $b\left(q_{0}\right), c\left(q_{0}\right) \in\left\{q_{0}\right\} \times \mathbb{R}$ of $F\left(q_{0}, \cdot\right)$ of indices 1,0 with $b\left(q_{0}\right)$ of index 1 
having a larger critical value. As $q$ increases further, the critical values of the critical points $a\left(q_{0}\right), b\left(q_{0}\right), c\left(q_{0}\right)$ of $F\left(q_{0}, \cdot\right)$ are traced out by $\pi_{q, z}(\Lambda) \cap\left\{q=q_{0}\right\}$. Eventually, the critical value of $b\left(q_{0}\right)$ is larger than the critical value of $a\left(q_{0}\right)$, and the critical value of $a\left(q_{0}\right)$ approaches the value of the critical value of $c\left(q_{0}\right)$. At the $q$-coordinate where the right cusp occurs, the critical points $a\left(q_{0}\right)$ and $c\left(q_{0}\right)$ merge to form a degenerate critical point which dies as $q$ increases further. After this point, $F\left(q_{0}, \cdot\right)$ is again a quadratic function of index 1 . After applying fiber preserving diffeomorphisms, $F$ will be quadratic at infinity of index 1. This procedure can be generalized to construct a g.q.i. function for a strand $L_{2}$ from a g.q.i. function for a strand $L_{1}$, when $L_{2}$ has an additional bubble resulting from a legendrian type $1^{ \pm}$move applied to $L_{1}$.

As can be seen from the previous example, there are choices in the domain and in the location of the critical points, but not in the critical values. If $\Lambda$ is defined by a g.q.i. function $F: S^{1} \times \mathbb{R}^{N} \rightarrow \mathbb{R}$, Theorem 3.4 shows that all other g.q.i. functions for $\Lambda$ arise from the following "natural modifications" of $F$ :

(1) Fiber Preserving Diffeomorphism Given a fiber preserving diffeomorphism $\Phi: S^{1} \times \mathbb{R}^{N} \rightarrow S^{1} \times \mathbb{R}^{N}$, consider $\widetilde{F}=F \circ \Phi$;

(2) Stabilization Let $Q: \mathbb{R}^{M} \rightarrow \mathbb{R}$ be a nondegenerate quadratic form, $Q(f)=\sum \alpha_{i j} f_{i} f_{j}$, and consider $\widetilde{F}: S^{1} \times \mathbb{R}^{N} \times \mathbb{R}^{M} \rightarrow \mathbb{R}$ defined by $\widetilde{F}(q, e, f)=F(q, e)+Q(f)$.

The following uniqueness theorem parallels a uniqueness result for quadratic at infinity generatating functions of lagrangian submanifolds, proved by Viterbo and Théret, [21],[16], [22]. The following can be proved by modifying Théret's careful proof to the legendrian setting, replacing references to Sikorav's lagrangian g.q.i. function existence results by the above mentioned legendrian g.q.i. function existence results, [3].

Uniqueness Theorem 3.4 (Théret) Let $\Lambda \subset \mathcal{J}^{1}\left(S^{1}\right)$ be legendrian isotopic to $j^{1}(0)$. If $F_{1}, F_{2}$ are both g.q.i. functions for $\Lambda$, then there exist nondegenerate quadratic forms $Q_{1}, Q_{2}$ and a fiber preserving diffeomorphism $\Phi$ so that $F_{2}+$ $Q_{2}=\left(F_{1}+Q_{1}\right) \circ \Phi$.

Definition 3.5 Consider a minimal legendrian link $L=\Lambda_{1} \amalg \Lambda_{0}$. Let

$$
F_{1}: S^{1} \times \mathbb{R}^{N_{1}} \rightarrow \mathbb{R}, \quad F_{0}: S^{1} \times \mathbb{R}^{N_{0}} \rightarrow \mathbb{R}
$$

be g.q.i. functions for $\Lambda_{1}, \Lambda_{0}$. Then the associated (quadratic at infinity) difference function of $L, \Delta: S^{1} \times \mathbb{R}^{N_{1}} \times \mathbb{R}^{N_{0}} \rightarrow \mathbb{R}$, is defined as

$$
\Delta\left(q, e_{1}, e_{0}\right):=F_{1}\left(q, e_{1}\right)-F_{0}\left(q, e_{0}\right) .
$$


Proposition 3.6 Suppose $L=\Lambda_{1} \amalg \Lambda_{0} \subset \mathcal{J}^{1}\left(S^{1}\right)$ is a minimal legendrian link. Then for any difference function $\Delta$ of $\Lambda_{1} \amalg \Lambda_{0}$, critical points of $\Delta$ are in $1--1$ correspondence with points $\left(\left(q_{0}, p_{0}, z_{1}\right),\left(q_{0}, p_{0}, z_{0}\right)\right) \in \Lambda_{1} \times \Lambda_{0}$, and 0 is never a critical value of $\Delta$.

Proof Using formula (3.1), it is easy to verify that the function $\Delta: S^{1} \times \mathbb{R}^{N_{1}} \times$ $\mathbb{R}^{N_{0}} \rightarrow \mathbb{R}$ generates the legendrian $D:=\left\{\left(q, p_{1}-p_{0}, z_{1}-z_{0}\right):\left(q, p_{i}, z_{i}\right) \in \Lambda_{i}\right\}$. Since critical points of $\Delta$ correspond to points where $D$ intersects $\{p=0\}$, there is a $1--1$ correspondence between critical points of $\Delta$ and the specified points of $\Lambda_{1} \times \Lambda_{0}$. Furthermore, since $\Lambda_{1} \cap \Lambda_{0}=\emptyset, 0$ cannot be a critical value for $\Delta$.

For $c \in \mathbb{R}$, a noncritical value of $\Delta: S^{1} \times \mathbb{R}^{N_{1}} \times \mathbb{R}^{N_{0}} \rightarrow \mathbb{R}$, let

$$
\Delta^{c}:=\left\{\left(q, e_{1}, e_{0}\right): \Delta\left(q, e_{1}, e_{0}\right) \leq c\right\} .
$$

For every link, there exists $M>0$ so that all critical values of $\Delta$ are contained in $[-M+\epsilon, M-\epsilon]$, for some $\epsilon>0$. For such an $M$, let

$$
\Delta^{ \pm \infty}:=\Delta^{ \pm M} .
$$

Definition 3.9 The total, positive, and negative homology groups of a minimal legendrian link $L=\Lambda_{1} \amalg \Lambda_{0} \subset \mathcal{J}^{1}\left(S^{1}\right)$ are defined as

$$
\begin{aligned}
H_{k}(L) & :=H_{k+q}\left(\Delta^{\infty}, \Delta^{-\infty}\right), \\
H_{k}^{+}(L) & :=H_{k+q}\left(\Delta^{\infty}, \Delta^{0}\right), \\
H_{k}^{-}(L) & :=H_{k+q}\left(\Delta^{0}, \Delta^{-\infty}\right), \quad k \in \mathbb{Z},
\end{aligned}
$$

where $\Delta$ is a difference function for $L, q$ is the index of $\Delta$, and the relative homology groups are calculated with coefficients in $\mathbb{Z}_{2}$.

From the definitions of these homology groups, if $\Delta$ has index $q$, then critical points of $\Delta$ of index $i$ will often contribute to the $H_{i-q}^{ \pm}(L), H_{i-q}(L)$. For this reason, when $\Delta$ is quadratic at infinity of index $q$, if $x$ is a critical point of $\Delta$ with index $i$, the shifted index of $x$ is defined as $i-q$.

The following is a classical result of Morse theory, but for the reader's convenience, a proof will be given. This lemma will be useful when showing that $H_{k}(L), H_{k}^{ \pm}(L)$ are well-defined invariants of $L$, and in the Section 6 calculations of these homology groups. 
Lemma 3.10 Consider a smooth 1-parameter family of quadratic at infinity functions $\Delta_{t}: S^{1} \times \mathbb{R}^{N} \rightarrow \mathbb{R}, t \in[0,1]$, of index $q$. Given paths $\alpha, \beta:[0,1] \rightarrow \mathbb{R}$ such that, for all $t, \alpha(t), \beta(t)$ are noncritical values of $\Delta_{t}$ with $\alpha(t)<\beta(t)$, then

$$
H_{q+k}\left(\Delta_{0}^{\beta(0)}, \Delta_{0}^{\alpha(0)}\right) \simeq H_{q+k}\left(\Delta_{t}^{\beta(t)}, \Delta_{t}^{\alpha(t)}\right), \quad \forall t \in[0,1], \quad \forall k \in \mathbb{Z} .
$$

Proof By applying fiber preserving diffeomorphisms, which will not change the calculation of the homology groups, it can be assumed that for all $t_{0}, t_{1} \in$ $[0,1], \Delta_{t_{0}}=\Delta_{t_{1}}$ outside a compact set. It suffices to show that for all $t_{0} \in$ $[0,1]$, there exists a neighborhood $U\left(t_{0}\right)$ of $t_{0}$ such that $H_{q+k}\left(\Delta_{t}^{\beta(t)}, \Delta_{t}^{\alpha(t)}\right) \simeq$ $H_{q+k}\left(\Delta_{t_{0}}^{\beta\left(t_{0}\right)}, \Delta_{t_{0}}^{\alpha\left(t_{0}\right)}\right)$.

First notice that since $\alpha(t)$ and $\beta(t)$ are noncritical values for $\Delta_{t}$, it is possible to choose $\epsilon>0$ such that for all $t \in[0,1]$, there are no critical values of $\Delta_{t}$ in $(\alpha(t)-2 \epsilon, \alpha(t)+2 \epsilon)$ or $(\beta(t)-2 \epsilon, \beta(t)+2 \epsilon)$. This implies that if $|b-\beta(t)|<2 \epsilon$ and $|a-\alpha(t)|<2 \epsilon$, then $H_{q+k}\left(\Delta_{t}^{b}, \Delta_{t}^{a}\right) \simeq H_{q+k}\left(\Delta_{t}^{\beta(t)}, \Delta_{t}^{\alpha(t)}\right)$.

Next, using such an $\epsilon$, choose a neighborhood $U\left(t_{0}\right)$ of $t_{0} \in[0,1]$ such that, for $t \in U\left(t_{0}\right)$,

(1) $\sup \left\{\left|\Delta_{t}(x)-\Delta_{t_{0}}(x)\right|: x \in S^{1} \times \mathbb{R}^{N}\right\}<\epsilon$, and

(2) $\left|\beta(t)-\beta\left(t_{0}\right)\right|<\epsilon$, and $\left|\alpha(t)-\alpha\left(t_{0}\right)\right|<\epsilon$.

By (2), $H_{q+k}\left(\Delta_{t}^{\beta(t)}, \Delta_{t}^{\alpha(t)}\right) \simeq H_{q+k}\left(\Delta_{t}^{\beta\left(t_{0}\right)+\epsilon}, \Delta_{t}^{\alpha\left(t_{0}\right)+\epsilon}\right)$. By (1), for $c=$ $\beta\left(t_{0}\right)$ and $c=\alpha\left(t_{0}\right)$, the inclusions $\Delta_{t}^{c-\epsilon} \subset \Delta_{t_{0}}^{c} \subset \Delta_{t}^{c+\epsilon} \subset \Delta_{t_{0}}^{c+2 \epsilon}$ induce homomorphisms

$$
\begin{aligned}
& H_{q+k}\left(\Delta_{t}^{\beta\left(t_{0}\right)-\epsilon}, \Delta_{t}^{\alpha\left(t_{0}\right)-\epsilon}\right) \stackrel{\phi_{1}}{\longrightarrow} H_{q+k}\left(\Delta_{t_{0}}^{\beta\left(t_{0}\right)}, \Delta_{t_{0}}^{\alpha\left(t_{0}\right)}\right) \stackrel{\phi_{2}}{\longrightarrow} \\
& H_{q+k}\left(\Delta_{t}^{\beta\left(t_{0}\right)+\epsilon}, \Delta_{t}^{\alpha\left(t_{0}\right)+\epsilon}\right) \stackrel{\phi_{3}}{\longrightarrow} H_{q+k}\left(\Delta_{t_{0}}^{\beta\left(t_{0}\right)+2 \epsilon}, \Delta_{t_{0}}^{\alpha\left(t_{0}\right)+2 \epsilon}\right) .
\end{aligned}
$$

Since $\left|\beta\left(t_{0}\right) \pm \epsilon-\beta(t)\right|,\left|\alpha\left(t_{0}\right) \pm \epsilon-\alpha(t)\right|,\left|\beta\left(t_{0}\right) \pm 2 \epsilon-\beta\left(t_{0}\right)\right|<2 \epsilon$, the first and third groups are isomorphic to $H_{q+k}\left(\Delta_{t}^{\beta(t)}, \Delta_{t}^{\alpha(t)}\right)$, the second and fourth groups are isomorphic to $H_{q+k}\left(\Delta_{t_{0}}^{\beta\left(t_{0}\right)}, \Delta_{t_{0}}^{\alpha\left(t_{0}\right)}\right)$, and $\phi_{2} \circ \phi_{1}$ and $\phi_{3} \circ \phi_{2}$ are isomorphisms. Thus $\phi_{2}$ is an isomorphism, and it follows that $H_{q+k}\left(\Delta_{t}^{\beta(t)}, \Delta_{t}^{\alpha(t)}\right) \simeq$ $H_{q+k}\left(\Delta_{t_{0}}^{\beta\left(t_{0}\right)}, \Delta_{t_{0}}^{\alpha\left(t_{0}\right)}\right)$.

Theorem 3.11 $H_{k}(L), H_{k}^{+}(L)$, and $H_{k}^{-}(L)$ are well-defined invariants of a minimal legendrian link $L \subset \mathcal{J}^{1}\left(S^{1}\right)$. 
Proof It must be shown that the homology groups do not depend on the choice of generating functions $F_{i}$ for the strands, and will not change as the link undergoes a legendrian isotopy.

Suppose $L=\Lambda_{1} \amalg \Lambda_{0}$, and let $F_{i}: S^{1} \times \mathbb{R}^{N_{i}} \rightarrow \mathbb{R}$ be g.q.i. function for $\Lambda_{i}$. It must be shown that if $\widetilde{F}_{i}: S^{1} \times \mathbb{R}^{M_{i}} \rightarrow \mathbb{R}$ are other g.q.i. function for $\Lambda_{i}$, then the relative homology groups of $\widetilde{\Delta}\left(q, \widetilde{e_{1}}, \widetilde{e_{0}}\right):=\widetilde{F}_{1}\left(q, \widetilde{e_{1}}\right)-\widetilde{F}_{0}\left(q, \widetilde{f_{0}}\right)$ agree, up to a shift of appropriate index, with those of $\Delta\left(q, e_{1}, e_{0}\right):=F_{1}\left(q, e_{1}, e_{0}\right)-$ $F_{0}\left(q, e_{1}, e_{0}\right)$. By the Uniqueness Theorem 3.4 , it is only necessary to check the cases where $\widetilde{\Delta}$ differs from $\Delta$ by a fiber preserving diffeomorphism or by a stabilization. If $\widetilde{\Delta}=\Delta \circ \Phi$, then the associated sublevel sets are diffeomorphic: $\widetilde{\Delta}^{c}=\Phi^{-1}\left(\Delta^{c}\right)$. Thus the relative homology groups are unchanged. Next, suppose that $\widetilde{\Delta}: S^{1} \times \mathbb{R}^{N} \times \mathbb{R}^{M} \rightarrow \mathbb{R}$ is defined by $\widetilde{\Delta}(q, e, f)=\Delta(q, e)+Q(f)$, where $\Delta$ is a g.q.i. function of index $q$, and $Q$ is a nondegenerate quadratic form of index $j$. It is easily verified that the critical values of $\Delta$ agree with those of $\tilde{\Delta}$, and that for a noncritical value $v$, for all $k \in \mathbb{Z}$, there is an isomorphism $\phi: C_{q+k}^{v}(\Delta) \rightarrow C_{q+j+k}^{v}(\widetilde{\Delta})$, where $C_{i}^{v}(\Delta)$ (respectively $C_{i}^{v}(\widetilde{\Delta})$ ) denotes the $i$-chains of $\Delta^{v}$ (respectively $\widetilde{\Delta}^{v}$ ). For all noncritical values $a<b$, and all $k \in \mathbb{Z}$, the isomorphism $\phi$ induces chain isomorphisms $\widetilde{\phi}: C_{q+k}^{b}(\Delta) / C_{q+k}^{a}(\Delta) \rightarrow$ $C_{q+j+k}^{b}(\widetilde{\Delta}) / C_{q+j+k}^{a}(\widetilde{\Delta})$, and thus $H_{q+k}\left(\Delta^{b}, \Delta^{a}\right) \simeq H_{q+j+k}\left(\widetilde{\Delta}^{b}, \widetilde{\Delta}^{a}\right)$, as desired.

Suppose $L_{t}, t \in[0,1]$, is a 1 -parameter family of minimal legendrian links. By Existence (3.2), there exist difference functions $\Delta_{t}: S^{1} \times \mathbb{R}^{N} \rightarrow \mathbb{R}$ of index $q$ for $L_{t}$. It must be shown that, for all $t \in[0,1]$, and all $k \in \mathbb{Z}$,

$$
\begin{aligned}
H_{k+q}\left(\Delta_{t}^{+\infty}, \Delta_{t}^{-\infty}\right) & \simeq H_{k+q}\left(\Delta_{0}^{+\infty}, \Delta_{0}^{-\infty}\right), \\
H_{k+q}\left(\Delta_{t}^{+\infty}, \Delta_{t}^{0}\right) & \simeq H_{k+q}\left(\Delta_{0}^{+\infty}, \Delta_{0}^{0}\right) \\
H_{k+q}\left(\Delta_{t}^{0}, \Delta_{t}^{-\infty}\right) & \simeq H_{k+q}\left(\Delta_{0}^{0}, \Delta_{0}^{-\infty}\right) .
\end{aligned}
$$

Choose paths $\alpha, \beta, \gamma:[0,1] \rightarrow \mathbb{R}$ such that $\alpha(t)$ is negative and is less than all critical values of $\Delta_{t}, \beta(t)=0$, and $\gamma(t)$ is positive and is greater than all critical values of $\Delta_{t}$. By construction and Proposition 3.6, $\alpha(t), \beta(t), \gamma(t)$ are noncritical values of $\Delta_{t}$, and thus the desired result holds by Lemma 3.10.

Proposition 3.12 For any minimal legendrian link $L, H_{k}(L) \simeq H_{k}\left(S^{1}\right)$, for all $k \in \mathbb{Z}$.

Proof By hypothesis, each strand of $L$ can be individually isotoped so that it is the graph of a function. Thus there exists a $1-$ parameter family of immersed legendrian links $L_{t}, t \in[0,1]$ with $L_{0}=L, L_{1}=j^{1}(+f) \amalg j^{1}(-f)$, where $j^{1}( \pm f)$ is the 1 -jet of $\pm f(q)= \pm(\cos (2 \pi q)+2)$. For the associated 1 -parameter family of difference functions $\Delta_{t}$ of $L_{t}$, choose paths $\alpha, \beta:[0,1] \rightarrow \mathbb{R}$ such that 
$\alpha(t)$ is negative and less than all critical values of $\Delta_{t}$, and $\beta(t)$ is positive and greater than all critical values of $\Delta_{t}$. Since it is easy to understand the total homology group of $L_{1}$, the desired result then follows by Lemma 3.10.

Lemma 3.13 For a function $\Delta: S^{1} \times \mathbb{R}^{N} \rightarrow \mathbb{R}$, and $a, b, c$ noncritical values of $\Delta$ with $a<b<c$, there is a long exact sequence

$$
\begin{aligned}
& \cdots \stackrel{\partial_{*}}{\rightarrow} H_{q+k}\left(\Delta^{b}, \Delta^{a}\right) \stackrel{i_{*}}{\rightarrow} H_{q+k}\left(\Delta^{c}, \Delta^{a}\right) \stackrel{\pi_{*}}{\rightarrow} \\
& H_{q+k}\left(\Delta^{c}, \Delta^{b}\right) \stackrel{\partial_{*}}{\rightarrow} H_{q+k-1}\left(\Delta^{b}, \Delta^{a}\right) \stackrel{i_{*}}{\rightarrow} \ldots .
\end{aligned}
$$

Proof Given $\Delta: S^{1} \times \mathbb{R}^{N} \rightarrow \mathbb{R}$ of index $q$, and a noncritical value $v$ of $\Delta$, $C_{k}^{v}(\Delta)$ will denote the $(q+k)$-chains of $\Delta^{v}$. Given a triple $a<b<c$ of noncritical values of $\Delta$, for each $k$, there is a natural exact sequence

$$
0 \rightarrow C_{k}^{b}(\Delta) / C_{k}^{a}(\Delta) \stackrel{i}{\rightarrow} C_{k}^{c}(\Delta) / C_{k}^{a}(\Delta) \stackrel{\pi}{\rightarrow} C_{k}^{c}(\Delta) / C_{k}^{b}(\Delta) \rightarrow 0 .
$$

Since $i, \pi$ are chain maps, it follows that there is an exact sequence

$$
\begin{aligned}
& \ldots \stackrel{\partial_{*}}{\rightarrow} H_{q+k}\left(\Delta^{b}, \Delta^{a}\right) \stackrel{i_{*}}{\rightarrow} H_{q+k}\left(\Delta^{c}, \Delta^{a}\right) \stackrel{\pi_{*}}{\rightarrow} \\
& H_{q+k}\left(\Delta^{c}, \Delta^{b}\right) \stackrel{\partial_{*}}{\rightarrow} H_{q+k-1}\left(\Delta^{b}, \Delta^{a}\right) \stackrel{i_{*}}{\rightarrow} \ldots .
\end{aligned}
$$

Corollary 3.14 For a minimal legendrian link $L$, there is an exact sequence

$$
\ldots \stackrel{\partial_{*}}{\rightarrow} H_{k}^{-}(L) \stackrel{i_{*}}{\rightarrow} H_{k}(L) \stackrel{\pi_{*}}{\rightarrow} H_{k}^{+}(L) \stackrel{\partial_{*}}{\rightarrow} H_{k-1}^{-}(L) \stackrel{i_{*}}{\rightarrow} \ldots
$$

Definition 3.15 For a minimal legendrian link $L$, form the positive and negative homology polynomials:

$$
\Gamma^{+}(\lambda)[L]=\sum_{k=-\infty}^{\infty} \operatorname{dim} H_{k}^{+}(L) \cdot \lambda^{k}, \quad \Gamma^{-}(\lambda)[L]=\sum_{k=-\infty}^{\infty} \operatorname{dim} H_{k}^{-}(L) \cdot \lambda^{k} .
$$

A comparison of $\Gamma^{+}(\lambda)[L]$ and $\Gamma^{-}(\lambda)[L]$ may detect that the legendrian link $L$ is ordered. Recall from Section 1 that polynomials $\alpha(\lambda)=\sum_{k=-\infty}^{\infty} a_{k} \lambda^{k}$ and $\beta(\lambda)=\sum_{k=-\infty}^{\infty} b_{k} \lambda^{k}$ are 1 -shift palindromic if $\alpha(\lambda)=\lambda \cdot \overline{\beta(\lambda)}$, where $\overline{\beta(\lambda)}$ denotes the palindrome of $\beta(\lambda)$.

Theorem 3.16 Let $L=\Lambda_{1} \amalg \Lambda_{0}$ be a minimal legendrian link, and let $\bar{L}$ denote its swap, $\bar{L}=\Lambda_{0} \amalg \Lambda_{1}$. Then $\Gamma^{+}(\lambda)[L]$ and $\Gamma^{-}(\lambda)[\bar{L}]$ are 1-shift palindromic, and $\Gamma^{-}(\lambda)[L]$ and $\Gamma^{+}(\lambda)[\bar{L}]$ are 1 -shift palindromic. 
Proof If $\Delta: S^{1} \times \mathbb{R}^{N-1} \rightarrow \mathbb{R}$ is a difference function for $L$, then $\bar{\Delta}=-\Delta$ is a difference function for $\bar{L}$. If $Q$ and $\bar{Q}$ denote the indices of $\Delta$ and $\bar{\Delta}$, then $\bar{Q}=N-1-Q$. Notice

$$
\begin{aligned}
H_{k}^{+}(L) & =H_{k+Q}\left(\Delta^{+\infty}, \Delta^{0}\right) \simeq H^{N-(k+Q)}\left(\Delta^{+\infty}, \Delta^{0}\right) \simeq H_{N-(k+Q)}\left(\bar{\Delta}^{0}, \bar{\Delta}^{-\infty}\right) \\
& =H_{N-(k+Q)-\bar{Q}}^{-}(\bar{L})=H_{N-(k+Q)-N+1+Q}^{-}(\bar{L})=H_{-k+1}^{-}(\bar{L}) .
\end{aligned}
$$

Thus $\operatorname{dim} H_{k}^{+}(L)=\operatorname{dim} H_{-k+1}^{-}(\bar{L})$, and it follows that $\Gamma^{+}(\lambda)[L]$ and $\Gamma^{-}(\lambda)[\bar{L}]$ are 1-shift palindromic. A similar calculation shows that $\Gamma^{-}(\lambda)[L]$ and $\Gamma^{+}(\lambda)[\bar{L}]$ are 1 -shift palindromic.

Corollary 3.17 Let $L=\Lambda_{1} \amalg \Lambda_{0}$ be a minimal legendrian link. If $\Gamma^{+}(\lambda)[L]$ and $\Gamma^{-}(\lambda)[L]$ are not 1 -shift palindromic, then the link $L$ is ordered.

Proof If $L$ is not ordered, then $\Gamma^{+}(\lambda)[L]=\Gamma^{+}(\lambda)[\bar{L}]$, and thus $\Gamma^{+}(\lambda)[L]$ and $\Gamma^{-}(\lambda)[L]$ must be 1 -shift palindromic.

\section{Algebraic Topology Tools}

In this section, some tools will be developed that will aid in the Section 6 calculations of the link polynomials of rational links and connect sums of rational links. The first result is called the Additive Extension Lemma since it gives conditions when, for $b_{2}>b_{1}>0$ and $a_{2}<a_{1}<0, \operatorname{dim} H_{*}\left(\Delta^{b_{2}}, \Delta^{0}\right)=\operatorname{dim} H_{*}\left(\Delta^{b_{2}}, \Delta^{b_{1}}\right)+$ $\operatorname{dim} H_{*}\left(\Delta^{b_{1}}, \Delta^{0}\right)$, and $\operatorname{dim} H_{*}\left(\Delta^{0}, \Delta^{a_{2}}\right)=\operatorname{dim} H_{*}\left(\Delta^{0}, \Delta^{a_{1}}\right)+\operatorname{dim} H_{*}\left(\Delta^{a_{1}}, \Delta^{a_{2}}\right)$.

Additive Extension Lemma 4.1 Suppose that $c_{1}^{ \pm}, c_{2}^{ \pm}$are critical values of a quadratic at infinity function $\Delta: S^{1} \times \mathbb{R}^{N} \rightarrow \mathbb{R}$ of index $q$, and that $a_{2}, a_{1}, b_{1}, b_{2}$ are noncritical values such that

$$
a_{2}<c_{2}^{-}<a_{1}<c_{1}^{-}<0<c_{1}^{+}<b_{1}<c_{2}^{+}<b_{2} .
$$

Suppose that

(1) $c_{2}^{+}$is the only critical value in $\left[b_{1}, b_{2}\right], c_{2}^{-}$is the only critical value in $\left[a_{2}, a_{1}\right]$, all critical points with values $c_{2}^{+}$are nondegenerate and have the same index, and all critical points with value $c_{2}^{-}$are nondegenerate and have the same index,

(2) $H_{q+k}\left(\Delta^{b_{1}}, \Delta^{a_{1}}\right)=0, \quad \forall k \in \mathbb{Z}$, and

(3) $H_{q+k}\left(\Delta^{0}, \Delta^{a_{1}}\right) \simeq H_{q+k+1}\left(\Delta^{b_{1}}, \Delta^{0}\right), \quad \forall k \in \mathbb{Z}$.

Then

$$
\begin{aligned}
& \operatorname{dim} H_{q+k}\left(\Delta^{b_{2}}, \Delta^{0}\right)=\operatorname{dim} H_{q+k}\left(\Delta^{b_{2}}, \Delta^{b_{1}}\right)+\operatorname{dim} H_{q+k}\left(\Delta^{b_{1}}, \Delta^{0}\right), \quad \text { and } \\
& \operatorname{dim} H_{q+k}\left(\Delta^{0}, \Delta^{a_{2}}\right)=\operatorname{dim} H_{q+k}\left(\Delta^{0}, \Delta^{a_{1}}\right)+\operatorname{dim} H_{q+k}\left(\Delta^{a_{1}}, \Delta^{a_{2}}\right), \quad \forall k \in \mathbb{Z} .
\end{aligned}
$$


Proof The statement about $\operatorname{dim} H_{q+k}\left(\Delta^{b_{2}}, \Delta^{0}\right)$ follows immediately if $\partial_{*} \equiv 0$ in the long exact sequence

$$
\begin{aligned}
& \ldots \stackrel{\partial_{*}}{\rightarrow} H_{q+k}\left(\Delta^{b_{1}}, \Delta^{0}\right) \stackrel{i_{*}}{\rightarrow} \\
& H_{q+k}\left(\Delta^{b_{2}}, \Delta^{0}\right) \stackrel{\pi_{*}}{\rightarrow} H_{q+k}\left(\Delta^{b_{2}}, \Delta^{b_{1}}\right) \stackrel{\partial_{*}}{\rightarrow} H_{q+k-1}\left(\Delta^{b_{1}}, \Delta^{0}\right) \stackrel{i_{*}}{\rightarrow} \ldots
\end{aligned}
$$

If there exists $k$ such that $0 \neq \operatorname{im} \partial_{*}\left(H_{q+k+1}\left(\Delta^{b_{2}}, \Delta^{b_{1}}\right)\right)$, then by hypothesis (1), $H_{q+k}\left(\Delta^{b_{2}}, \Delta^{b_{1}}\right)=0$, and thus

$$
\operatorname{dim} H_{q+k}\left(\Delta^{b_{1}}, \Delta^{0}\right)>\operatorname{dim} H_{q+k}\left(\Delta^{b_{2}}, \Delta^{0}\right) .
$$

Since all critical points with values in $\left[b_{1}, b_{2}\right]$ have index $q+k+1$, another exact sequence argument, using the fact that $H_{*}\left(\Delta^{b_{1}}, \Delta^{a_{1}}\right)=0$ for all $*$, proves

$$
H_{q+k-1}\left(\Delta^{b_{2}}, \Delta^{a_{1}}\right)=0 .
$$

The facts 4.1.2 and 4.1.3 combine with the hypotheses

$$
\operatorname{dim} H_{q+k-1}\left(\Delta^{0}, \Delta^{a_{1}}\right)=\operatorname{dim} H_{q+k}\left(\Delta^{b_{1}}, \Delta^{0}\right)
$$

to give a contradiction to the necessary surjectivity of $\partial_{*}$ in the exact sequence (4.1.4)

$$
\ldots \stackrel{\pi_{*}}{\rightarrow} H_{q+k}\left(\Delta^{b_{2}}, \Delta^{0}\right) \stackrel{\partial_{*}}{\rightarrow} H_{q+k-1}\left(\Delta^{0}, \Delta^{a_{1}}\right) \stackrel{i_{*}}{\rightarrow} H_{q+k-1}\left(\Delta^{b_{2}}, \Delta^{a_{1}}\right) \stackrel{\pi_{*}}{\rightarrow} \ldots
$$

This proves the claim about $\operatorname{dim} H_{q+k}\left(\Delta^{b_{2}}, \Delta^{0}\right)$. A proof of the claim about $\operatorname{dim} H_{q+k}\left(\Delta^{0}, \Delta^{a_{2}}\right)$ can be proved similarly.

The following proposition will be useful when calculating the homology polynomials of legendrian links that are topologically the rational links $q$, for $q \geq 1$.

Positive Integral Proposition 4.2 Suppose the minimal legendrian link $L=\Lambda_{1} \amalg \Lambda_{0}$ has a difference function $\Delta: S^{1} \times \mathbb{R}^{N} \rightarrow \mathbb{R}$ with critical values $c_{1}^{ \pm}, \ldots, c_{n}^{ \pm}$, and noncritical values $a_{1}, \ldots, a_{n}, b_{n}, \ldots, b_{1}$ satisfying

$$
\begin{aligned}
& a_{1}<c_{1}^{-}<a_{2}<c_{2}^{-}<\cdots<a_{n-1}<c_{n-1}^{-}<a_{n}<c_{n}^{-}<0, \\
& 0<c_{n}^{+}<b_{n}<c_{n-1}^{+}<b_{n-1}<\cdots<c_{2}^{+}<b_{2}<c_{1}^{+}<b_{1} .
\end{aligned}
$$

If

(1) for $k=1, \ldots, n$, there are $h_{k}$ nondegenerate critical points with value $c_{k}^{+}$, and $h_{k}$ nondegenerate critical points with value $c_{k}^{-}$; all critical points with value $c_{k}^{+}$have shifted index $i_{k}+1$, and all critical points with value $c_{k}^{-}$have shifted index $i_{k}$; and

(2) for $k=2, \ldots, n, H_{*}\left(\Delta^{b_{k}}, \Delta^{a_{k}}\right)=0$, for all $* \in \mathbb{Z}$,

then

$$
\Gamma^{+}(\lambda)[L]=\sum_{k=1}^{n} h_{k} \lambda^{i_{k}+1}, \quad \Gamma^{-}(\lambda)[L]=\sum_{k=1}^{n} h_{k} \lambda^{i_{k}} .
$$


Proof By hypothesis (1),

$$
\begin{gathered}
\operatorname{dim} H_{q+*}\left(\Delta^{0}, \Delta^{a_{n}}\right)=\operatorname{dim} H_{q+*+1}\left(\Delta^{b_{n}}, \Delta^{0}\right)=\left\{\begin{array}{ll}
h_{n} & *=i_{n} \\
0 & \text { else }
\end{array},\right. \text { and } \\
\operatorname{dim} H_{q+*}\left(\Delta^{a_{k+1}}, \Delta^{a_{k}}\right)=\operatorname{dim} H_{q+*+1}\left(\Delta^{b_{k}}, \Delta^{b_{k+1}}\right)= \begin{cases}h_{k} & *=i_{k} \\
0 & \text { else }\end{cases}
\end{gathered}
$$

for $k=1, \ldots, n-1$. Thus it suffices to show that for $n-1 \geq k \geq 1$,

$\operatorname{dim} H_{q+*}\left(\Delta^{b_{k}}, \Delta^{0}\right)=\operatorname{dim} H_{q+*}\left(\Delta^{b_{k+1}}, \Delta^{0}\right)+\operatorname{dim} H_{q+*}\left(\Delta^{b_{k}}, \Delta^{b_{k+1}}\right)$, and $\operatorname{dim} H_{q+*}\left(\Delta^{0}, \Delta^{a_{k}}\right)=\operatorname{dim} H_{q+*}\left(\Delta^{0}, \Delta^{a_{k+1}}\right)+\operatorname{dim} H_{q+*}\left(\Delta^{a_{k+1}}, \Delta^{a_{k}}\right), \forall * \in \mathbb{Z}$.

This will be proven by repeatedly applying the Additive Extension Lemma 4.1. To apply this lemma, it must be shown that for $n \geq k \geq 2$,

$$
H_{q+*}\left(\Delta^{0}, \Delta^{a_{k}}\right) \simeq H_{q+*+1}\left(\Delta^{b_{k}}, \Delta^{0}\right) .
$$

As mentioned above, this is true when $k=n$. Assume it is true when $k=\ell$. Then the Additive Extension Lemma applied to

$$
a_{\ell-1}<c_{\ell-1}^{-}<a_{\ell}<c_{\ell}^{-}<0<c_{\ell}^{+}<b_{\ell}<c_{\ell-1}^{+}<b_{\ell-1}
$$

shows that

$$
\begin{aligned}
\operatorname{dim} H_{q+*+1}\left(\Delta^{b_{\ell-1}}, \Delta^{0}\right) & =\operatorname{dim} H_{q+*+1}\left(\Delta^{b_{\ell}}, \Delta^{0}\right)+\operatorname{dim} H_{q+*+1}\left(\Delta^{b_{\ell-1}}, \Delta^{b_{\ell}}\right) \\
& =\operatorname{dim} H_{q+*}\left(\Delta^{0}, \Delta^{a_{\ell}}\right)+\operatorname{dim} H_{q+*}\left(\Delta^{a_{\ell}}, \Delta^{a_{\ell-1}}\right) \\
& =\operatorname{dim} H_{q+*}\left(\Delta^{0}, \Delta^{a_{\ell-1}}\right) .
\end{aligned}
$$

Thus the isomorphism holds when $k=\ell-1$. This completes the proof.

The next proposition will be used to calculate the homology polynomials of minimal legendrian links that are topologically the rational links $q$, for $q<1$.

Zero Integral Proposition 4.3 Suppose the minimal legendrian link $L=$ $\Lambda_{1} \amalg \Lambda_{0}$ has a difference function $\Delta: S^{1} \times \mathbb{R}^{N} \rightarrow \mathbb{R}$ with critical values $c_{1}^{0}, c_{1}^{1}, c_{2}^{ \pm}$, $\ldots, c_{n}^{ \pm}$and noncritical values $a_{2}, \ldots, a_{n}, b_{n}, \ldots, b_{1}$ satisfying

$$
\begin{aligned}
& a_{2}<c_{2}^{-}<\cdots<a_{n-1}<c_{n-1}<a_{n}<c_{n}^{-}<0 \\
& 0<c_{n}^{+}<b_{n}<c_{n-1}^{+}<b_{n-1}<\cdots<c_{2}^{+}<b_{2}<c_{1}^{0}<c_{1}^{1}<b_{1} .
\end{aligned}
$$

If, for $k=2, \ldots, n$,

(1) there are $h_{k}$ nondegenerate critical points with value $c_{k}^{+}$, and $h_{k}$ nondegenerate critical points with value $c_{k}^{-}$; all critical points with value $c_{k}^{+}$ have shifted index $i_{k}+1$, while all critical points with value $c_{k}^{-}$have shifted index $i_{k}$, and 
(2) $H_{*}\left(\Delta^{b_{k}}, \Delta^{a_{k}}\right)=0$, for all $* \in \mathbb{Z}$,

then

$$
\Gamma^{+}(\lambda)[L]=(1+\lambda)+\sum_{k=2}^{n} h_{k} \lambda^{i_{k}+1}, \quad \Gamma^{-}(\lambda)[L]=\sum_{k=2}^{n} h_{k} \lambda^{i_{k}}
$$

Proof Using the hypothesis $H_{*}\left(\Delta^{b_{k}}, \Delta^{a_{k}}\right)=0$ for $k=3, \ldots, n$, arguments as in the proof of Proposition 4.2 prove that

$$
\begin{aligned}
\Gamma^{-}(\lambda)[L] & =\operatorname{dim} H_{q+i_{n}}\left(\Delta^{0}, \Delta^{a_{n}}\right) \lambda^{i_{n}}+\operatorname{dim} H_{q+i_{n-1}}\left(\Delta^{a_{n}}, \Delta^{a_{n-1}}\right) \lambda^{i_{n-1}}+\ldots \\
& +\operatorname{dim} H_{q+i_{2}}\left(\Delta^{a_{3}}, \Delta^{a_{2}}\right) \lambda^{i_{2}} \\
& =\sum_{k=2}^{n} h_{k} \lambda^{i_{k}} .
\end{aligned}
$$

To prove the claim about $\Gamma^{+}(\lambda)[L]$, it suffices to show that

$$
\operatorname{dim} H_{k}^{+}(L)= \begin{cases}\operatorname{dim} H_{k-1}^{-}(L)+1, & k=0,1 \\ \operatorname{dim} H_{k-1}^{-}(L), & \text { else. }\end{cases}
$$

By Proposition 3.12, $\operatorname{dim} H_{k}(L)=1$, when $k=0,1$, and vanishes otherwise. Thus the desired calculations of $H_{k}^{+}(L)$ will follow if it is shown that $i_{*}=0$ in the exact sequence

$$
\ldots \stackrel{\partial_{*}}{\rightarrow} H_{k}^{-}(L) \stackrel{i_{*}}{\rightarrow} H_{k}(L) \stackrel{\pi_{*}}{\rightarrow} H_{k}^{+}(L) \stackrel{\partial_{*}}{\rightarrow} H_{k-1}^{-}(L) \stackrel{i_{*}}{\rightarrow} \ldots
$$

The map $i_{*}$ is induced by the inclusion map $i: \frac{C_{k}^{0}(\Delta)}{C_{k}^{a_{2}}(\Delta)} \rightarrow \frac{C_{k}^{b_{1}}(\Delta)}{C_{k}^{a_{2}}(\Delta)}$. Since $i=$ $i_{2} \circ i_{1}$, where

$$
\frac{C_{k}^{0}(\Delta)}{C_{k}^{a_{2}}(\Delta)} \stackrel{i_{1}}{\rightarrow} \frac{C_{k}^{b_{2}}(\Delta)}{C_{k}^{a_{2}}(\Delta)} \stackrel{i_{2}}{\rightarrow} \frac{C_{k}^{b_{1}}(\Delta)}{C_{k}^{a_{2}}(\Delta)}
$$

$i_{*}=\left(i_{2}\right)_{*} \circ\left(i_{1}\right)_{*}$. However, since $H_{*}\left(\Delta^{b_{2}}, \Delta^{a_{2}}\right)=0,\left(i_{1}\right)_{*}=0$, and thus $i_{*}=0$, as desired.

\section{Index Calculations}

Propositions 4.2 and 4.3 will make it easy to calculate the homology polynomials of a minimal legendrian link $L=\Lambda_{1} \amalg \Lambda_{0}$ from a difference function that is in a "nice" form. To apply the propositions, it will be necessary to find the critical points of $\Delta$, and calculate the indices of these critical points. Critical points of $\Delta$ are easily described in terms of $\Lambda_{1} \amalg \Lambda_{0}$ : as was shown in Proposition 
3.6, they correspond to points $\left(\left(q_{0}, p_{0}, z_{1}\right),\left(q_{0}, p_{0}, z_{0}\right)\right) \in \Lambda_{1} \times \Lambda_{0}$. The indices of the critical points of $\Delta$ can easily be calculated in terms of data from the $(q, z)$-projection of the link: as will be shown in Proposition 5.5, the index of the critical point corresponding to $\left(\left(q_{0}, p_{0}, z_{1}\right),\left(q_{0}, p_{0}, z_{0}\right)\right)$ can be calculated as a difference of "branch indices" of $\left(q_{0}, p_{0}, z_{1}\right)$ and of $\left(q_{0}, p_{0}, z_{0}\right)$, and a Morse "graph index" between $\left(q_{0}, p_{0}, z_{1}\right)$ and $\left(q_{0}, p_{0}, z_{0}\right)$.

Throughout this section, $\pi_{q, z}, \pi_{q, p}: \mathcal{J}^{1}\left(S^{1}\right) \rightarrow S^{1} \times \mathbb{R}$ will denote the projections to the $(q, z)-,(q, p)$-coordinates, respectively.

Definition 5.1 Given a legendrian knot $\Lambda \subset \mathcal{J}^{1}\left(S^{1}\right)$, the branches of $\Lambda$ are the connected components of $\Lambda \backslash C$, where $C$ denotes the set of points that project to cusp points in $\pi_{q, z}(\Lambda)$. Branches $V_{1}, V_{0}$ of $\Lambda$ are adjacent if their closures $\bar{V}_{i}$ intersect. If $V_{1}, V_{0}$ are adjacent, $V_{1}>V_{0}$ if there exists $v \in \bar{V}_{1} \cap \bar{V}_{0}$ and a path $\gamma \subset \pi_{q, z}(\Lambda)$ with $\gamma[0,1 / 2) \subset \pi_{q, z}\left(V_{0}\right), \gamma(1 / 2)=\pi_{q, z}(v)$, and $\gamma(1 / 2,1] \subset \pi_{q, z}\left(V_{1}\right)$, so that $\pi_{q, z}(v)$ is an up cusp along the path.

Definition 5.2 Suppose $\Lambda \subset \mathcal{J}^{1}\left(S^{1}\right)$ is legendrian isotopic to $j^{1}(0)$. Let $\left\{V_{i}\right\}$ denote the set of branches of $\Lambda$. Suppose there exists a branch $\mathcal{I} \in\left\{V_{i}\right\}, v \in \mathcal{I}$, and a contact isotopy $\kappa_{t}, t \in[0,1]$, so that $\kappa_{1}(\Lambda)=j^{1}(0)$, and $\pi_{q, z}\left(\kappa_{t}(v)\right)$ is never a cusp. Then the branch index $i_{B}:\left\{V_{i}\right\} \rightarrow \mathbb{Z}$ is defined by $i_{B}\left(V_{i}\right)=0$, if $V_{i}=\mathcal{I}$, and $i_{B}\left(V_{1}\right)-i_{B}\left(V_{0}\right)=1$, if $V_{1}, V_{0}$ are adjacent with $V_{1}>V_{0}$.

From this definition, it appears that the above branch index may depend on the choice of an initial branch $\mathcal{I}$. However, the next proposition shows that this is not the case.

Proposition 5.3 Suppose $\Lambda$ is legendrian isotopic to $j^{1}(0), V$ is a branch of $\Lambda$, and $v \in V$. If $F: S^{1} \times \mathbb{R}^{N} \rightarrow \mathbb{R}$ is a g.q.i.function of index $Q$ for $\Lambda$, and $\left(q_{v}, e_{v}\right) \in S^{1} \times \mathbb{R}^{N}$ corresponds to $v$, then $i_{B}(V)=\operatorname{ind} F\left(q_{v}, \cdot\right)\left(e_{v}\right)-Q$.

Proof Let $\Lambda_{t}$ be a legendrian isotopy with $\Lambda_{0}=j^{1}(0), \Lambda_{1}=\Lambda$, and let $\pi_{q, p}\left(\Lambda_{t}\right)$ denote the lagrangian projections. By a classical result, see for example Appendix B of [17] or [18], the shifted index of the fiber function $F\left(q_{v}, \cdot\right)\left(e_{v}\right)-$ $Q$ is equal to the Maslov index of a path $\gamma(t) \in \pi_{q, p}\left(\Lambda_{t}\right), t \in[0,1]$, with $\gamma(1)=\pi_{q, p}(v)$. By a morsification procedure, see for example [11], the rotation calculations necessary to calculate the Maslov index can be computed in terms of cusps: by an appropriate choice of isotopy, the Maslov index equals the difference between the number of up and down cusps along a path $\pi_{q, z}\left(\Lambda_{1}\right)$ that starts at $\pi_{q, z}(\iota), \iota \in \mathcal{I}$, and ends at $\pi_{q, z}(v)$. 
Definition 5.4 Given legendrian knots $\Lambda_{1}, \Lambda_{0} \subset \mathcal{J}^{1}\left(S^{1}\right)$, suppose $\left(q_{0}, p_{0}, z_{1}\right)$ $\in V_{1},\left(q_{0}, p_{0}, z_{0}\right) \in V_{0}$, where $V_{1}, V_{0}$ are branches of $\Lambda_{1}, \Lambda_{0}$, respectively. Then there exist functions $f_{1}, f_{0}: U \rightarrow \mathbb{R}$ such that near $\left(q_{0}, p_{0}, z_{1}\right), \pi_{q, z}\left(V_{1}\right)=$ $\left\{\left(q, f_{1}(q)\right)\right\}$, and near $\left(q_{0}, p_{0}, z_{0}\right), \pi_{q, z}\left(V_{0}\right)=\left\{\left(q, f_{0}(q)\right)\right\}$. It follows that $q_{0}$ is a critical point of $f_{1}-f_{0}$. Then $\left(\left(q_{0}, p_{0}, z_{1}\right),\left(q_{0}, p_{0}, z_{1}\right)\right) \in \Lambda_{1} \times \Lambda_{0}$ is nondegenerate if $q_{0}$ is a nondegenerate critical point of $f_{1}-f_{0}$; the graph index, $i_{\Gamma}$, of a nondegenerate point is the Morse index of $f_{1}-f_{0}$ at $q_{0}$.

Proposition 5.5 Suppose $L=\Lambda_{1} \amalg \Lambda_{0} \subset \mathcal{J}^{1}\left(S^{1}\right)$ is a minimal legendrian link. Given a nondegenerate $\left(\left(q_{0}, p_{0}, z_{1}\right),\left(q_{0}, p_{0}, z_{0}\right)\right) \in \Lambda_{1} \times \Lambda_{0}$, the corresponding nondegenerate critical point $x$ of $\Delta$ has shifted index $\tilde{i}(x)$ given by

$$
\tilde{i}(x)=i_{B}\left(V_{1}\right)-i_{B}\left(V_{0}\right)+i_{\Gamma}\left(\left(q_{0}, p_{0}, z_{1}\right),\left(q_{0}, p_{0}, z_{0}\right)\right),
$$

where $\left(q_{0}, p_{0}, z_{i}\right)$ lies in the branch $V_{i}$ of $\Lambda_{i}$.

Proof Suppose that $x=\left(q_{0}, e_{1}, e_{0}\right)$ is a critical point of $\Delta$. By fiber preserving diffeomorphisms, it can be assumed that in a neighborhood $U$ of $\left(q_{0}, e_{1}, e_{0}\right)$,

$\left.\Delta\left(q, \xi_{1}, \xi_{0}\right)\right|_{U}=\left.\left(F_{1}\left(q, \xi_{1}\right)-F_{0}\left(q, \xi_{0}\right)\right)\right|_{U}=\left(G_{1}(q)+J\left(\xi_{1}\right)\right)-\left(G_{0}(q)+H\left(\xi_{0}\right)\right)$,

and thus it suffices to show

$$
\text { ind } \Delta(x)-\operatorname{ind} \Delta=\operatorname{ind}\left(G_{1}-G_{0}\right)\left(q_{0}\right)+\text { ind } J\left(e_{1}\right)+\operatorname{ind}(-H)\left(e_{0}\right) \text {. }
$$

It is easy to verify that

$$
\operatorname{ind}\left(G_{1}-G_{0}\right)\left(q_{0}\right)=i_{\Gamma}\left(\left(q_{0}, p_{0}, z_{1}\right),\left(q_{0}, p_{0}, z_{0}\right)\right) .
$$

Let $-\Lambda_{0}:=\left\{(q,-p,-z):(q, p, z) \in \Lambda_{0}\right\}$. Then $-F_{0}$ is a g.q.i. function for $-\Lambda_{0}$ and $\left(q_{0},-p_{0},-z_{0}\right)$ lies in a branch $W_{0}$ of $-\Lambda_{0}$ with $i_{B}\left(W_{0}\right)=-i_{B}\left(V_{0}\right)$. Since $\left(q_{0}, e_{1}\right) \in S^{1} \times \mathbb{R}^{N_{1}}$ corresponds to $\left(q_{0}, p_{0}, z_{1}\right) \in V_{1}$, and $\left(q_{0}, e_{0}\right) \in S^{1} \times \mathbb{R}^{N_{0}}$ corresponds to $\left(q_{0},-p_{0},-z_{0}\right) \in W_{0}$, Proposition 5.3 implies that

$$
\text { ind } J\left(e_{1}\right)-i_{Q_{1}}=i_{B}\left(V_{1}\right), \quad \text { ind }(-H)\left(e_{0}\right)-i_{Q_{0}}=i_{B}\left(W_{0}\right)=-i_{B}\left(V_{0}\right) \text {, }
$$

where $i_{Q_{1}}$ is the index of $J\left(e_{1}\right), i_{Q_{0}}$ is the index of $-H\left(e_{0}\right)$. Since ind $\Delta=$ $i_{Q_{1}}+i_{Q_{0}}$, the desired result follows.

\section{Polynomial Calculations}

In this section, the positive and negative polynomials are calculated for the standard rational legendrian links, flypes of these standard links, and for connect sums of these flypes. 
Theorem 6.1 Consider the legendrian link $L=\left(2 h_{n}, v_{n-1}, \ldots, v_{1}, 2 h_{1}\right)$. Then

$$
\begin{aligned}
& \Gamma^{-}(\lambda)[L]=h_{1}+h_{2} \lambda^{-v_{1}}+h_{3} \lambda^{-v_{1}-v_{2}}+\cdots+h_{n} \lambda^{-v_{1}-v_{2}-\cdots-v_{n-1}}, \\
& \Gamma^{+}(\lambda)[L]= \begin{cases}\lambda \cdot \Gamma^{-}(\lambda)[L], & h_{1} \geq 1 \\
(1+\lambda)+\lambda \cdot \Gamma^{-}(\lambda)[L], & h_{1}=0 .\end{cases}
\end{aligned}
$$

Proof First consider the case of $h_{1} \geq 1$. It is possible to legendrian isotop $L$ to a position so that it has a difference function $\Delta: S^{1} \times \mathbb{R}^{N} \rightarrow \mathbb{R}$ with $2 h_{1}+\cdots+2 h_{n}$ nondegenerate critical points: for $i=1, \ldots, n, \Delta$ has $h_{i}$ critical points with, by Proposition 5.5, shifted index $-v_{1}-v_{2}-\cdots-v_{i-1}+1$ and value $c_{i}^{+}$, and $h_{i}$ critical points of shifted index $-v_{1}-v_{2}-\cdots-v_{i-1}$ and value $c_{i}^{-}$, where

$$
c_{1}^{-}<c_{2}^{-}<\cdots<c_{n}^{-}<0<c_{n}^{+}<\cdots<c_{2}^{+}<c_{1}^{+} .
$$

Figure 6.1.1 illustrates one such construction for the link $(2,2,4,1,2)$.

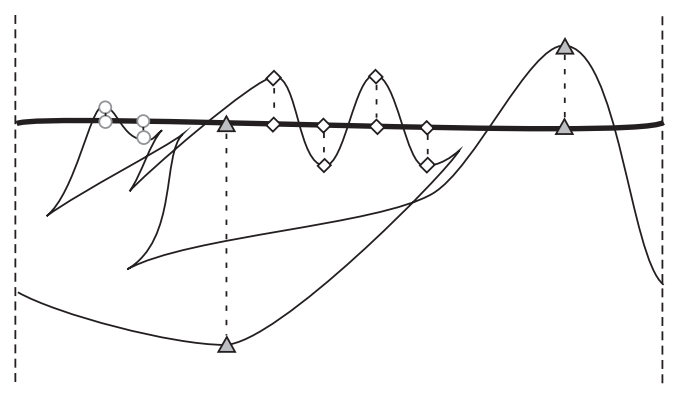

Figure 6.1.1: The legendrian link $(2,2,4,1,2)$ positioned so that it has a difference function $\Delta$ with $(2+4+2)$ critical points which are represented as pairs of points $\left(\left(q_{0}, p_{0}, z_{1}\right),\left(q_{0}, p_{0}, z_{0}\right)\right) \in \Lambda_{1} \times \Lambda_{0}$. The shifted indices of these critical points are calculated by Proposition 5.5.

For $n \geq k \geq 2$, choose $a_{k}, b_{k}$ so that

$$
c_{k-1}^{-}<a_{k}<c_{k}^{-}<0<c_{k}^{+}<b_{k}<c_{k-1}^{+} .
$$

The claimed calculations will follow immediately from the Positive Integral Proposition 4.2 if it is shown that $H_{*}\left(\Delta^{b_{k}}, \Delta^{a_{k}}\right)=0$, for all $* \in \mathbb{Z}$. By applying a deformation argument as in the proof of Proposition 3.12, it is possible to construct a 1-parameter family of quadratic at infinity functions $\Delta_{t}: S^{1} \times \mathbb{R}^{N} \rightarrow \mathbb{R}$ such that $a_{k}, b_{k}$ are noncritical values of $\Delta_{t}$ for all $t \in[0,1]$, where $\Delta_{0}=\Delta$, and $\Delta_{1}$ has no critical points in $\left[a_{k}, b_{k}\right]$. Thus by Lemma 3.10, 
$H_{*}\left(\Delta_{0}^{b_{k}}, \Delta_{0}^{a_{k}}\right)=H_{*}\left(\Delta_{1}^{b_{k}}, \Delta_{1}^{a_{k}}\right)=0$, for all $* \in \mathbb{Z}$. This completes the proof in the case of $h_{1} \geq 1$.

In the case of $h_{1}=0$, it is possible to legendrian isotop $L$ to a position so that it has a difference function $\Delta: S^{1} \times \mathbb{R}^{N} \rightarrow \mathbb{R}$ that has 1 critical point of shifted index 0 with value $c_{1}^{0}, 1$ critical point of shifted index 1 with value $c_{1}^{1}$, and for $i=2, \ldots, n, h_{i}$ critical points of shifted index $-v_{1}-v_{2}-\cdots-v_{i-1}$ with value $c_{i}^{-}$, and $h_{i}$ critical points of index $-v_{1}-v_{2}-\cdots-v_{i-1}+1$ with value $c_{i}^{+}$, where

$$
c_{2}^{-}<\cdots<c_{n}^{-}<0<c_{n}^{+}<\cdots<c_{2}^{+}<c_{1}^{0}<c_{1}^{1} .
$$

For $k=2, \ldots, n$, choose $a_{k}, b_{k}$ so that

$$
a_{2}<c_{2}^{-}<c_{2}^{+}<b_{2}<c_{1}^{0}, \quad c_{k-1}^{-}<a_{k}<c_{k}^{-}<0<c_{k}^{+}<b_{k}<c_{k-1}^{+}, n \geq k \geq 3 .
$$

An argument as in the above paragraph proves $H_{*}\left(\Delta^{b_{k}}, \Delta^{a_{k}}\right)=0$ for all $* \in \mathbb{Z}$, for $k=2, \ldots, n$. Thus the Zero Integral Proposition 4.3 gives the desired calculation of $\Gamma^{+}(\lambda)[L]$ and $\Gamma^{-}(\lambda)[L]$.

Next, the positive and negative polynomials will be calculated for horizontal flypes of a standard rational link. Recall the notation $\left(2 h_{n}, v_{n-1}, 2 h_{n-1}^{p_{n-1}}, \ldots, v_{1}\right.$, $\left.2 h_{1}^{p_{1}}\right), p_{i} \in\left\{0, \ldots, 2 h_{i}\right\}$, introduced in (1.11).

Theorem 6.2 Consider the legendrian link $L=\left(2 h_{n}, v_{n-1}, 2 h_{h-1}^{p_{n-1}}, \ldots, v_{1}\right.$, $\left.2 h_{1}^{p_{1}}\right)$. For $j=2, \ldots, n-1$, let $\sigma(j)=1+\sum_{i=1}^{j} p_{i} \bmod 2$. Then

$$
\begin{aligned}
& \Gamma^{-}(\lambda)[L]=h_{1}+\sum_{i=2}^{n} h_{i} \lambda^{(-1)^{\sigma(1)} v_{1}+(-1)^{\sigma(2)} v_{2}+\cdots+(-1)^{\sigma(i-1)} v_{i-1},} \\
& \Gamma^{+}(\lambda)[L]= \begin{cases}\lambda \cdot \Gamma^{-}(\lambda)[L], & h_{1} \geq 1 \\
(1+\lambda)+\lambda \cdot \Gamma^{-}(\lambda)[L], & h_{1}=0 .\end{cases}
\end{aligned}
$$

Proof The claim will follow using arguments as in the proof of Theorem 6.1 if it is shown that for $L=\Lambda_{1} \amalg \Lambda_{0}=\left(2 h_{n}, v_{n-1}, 2 h_{h-1}^{p_{n-1}}, \ldots, v_{1}, 2 h_{1}^{p_{1}}\right)$, there exists a difference function $\Delta$ with $2 h_{1}+2 h_{2}+\cdots+2 h_{n}$ nondegenerate critical points, where for $k=2, \ldots, n$, the $2 h_{k}$ critical points correspond to $2 h_{k}$ pairs of points on branches $W_{1}^{k} \subset \Lambda_{1}, W_{0}^{k} \subset \Lambda_{0}$ with branch indices

$$
i_{B}\left(W_{1}^{k}\right)-i_{B}\left(W_{0}^{k}\right)=(-1)^{\sigma(1)} v_{1}+(-1)^{\sigma(2)} v_{2}+\cdots+(-1)^{\sigma(k-1)} v_{k-1},
$$

for $\sigma(j)=1+\sum_{i=1}^{j} p_{i} \bmod 2$. As seen in the proof of Theorem 6.1, there exists such a difference function for $L_{0}=\left(2 h_{n}, v_{n-1}, 2 h_{h-1}^{p_{n-1}}, \ldots, v_{1}, 2 h_{1}^{p_{1}}\right)$, when $p_{k}=$ 0 for all $k$. For arbitrary $p_{n-1}, \ldots, p_{1}$, assume there exists such a difference function for $L=\left(2 h_{n}, v_{n-1}, 2 h_{h-1}^{p_{n-1}}, \ldots, v_{1}, 2 h_{1}^{p_{1}}\right)$. Let $\sigma(\ell)=1+\sum_{k=1}^{\ell} p_{k}$ 
mod 2. Consider $L^{\prime}$ which differs from $L$ by one additional horizontal flype: $L^{\prime}=\left(2 h_{n}, v_{n-1}, 2 h_{h-1}^{w_{n-1}}, \ldots, v_{1}, 2 h_{1}^{w_{1}}\right), \exists j: w_{k}=p_{k}$, for $k \neq j$, and $w_{j}=$ $p_{j}+1$. Notice that

$$
\sigma^{\prime}(\ell):=1+\sum_{k=1}^{\ell} w_{k} \quad \bmod 2 \equiv_{2} \begin{cases}\sigma(\ell), & \ell \leq j-1 \\ \sigma(\ell)+1, & \ell \geq j .\end{cases}
$$

As in the case of $L$, there exists a difference function $\Delta^{\prime}$ for $L^{\prime}$ with $2 h_{1}+\cdots+$ $2 h_{n}$ critical points; however, now, due of the flype in the $h_{j}$ term, there is a change in the indices of the branches containing points in the pairs associated to the terms $2 h_{j+1}, \ldots, 2 h_{n}$. Figure 6.2.1 illustrates such a construction for horizontal flypes of $(2,1,2,2,2)$. More precisely, for $k=2, \ldots, n$, the $2 h_{k}$ critical points are associated to points on branches $Y_{1}^{k} \subset \Lambda_{1}^{\prime}, Y_{0}^{k} \subset \Lambda_{0}^{\prime}$ with branch indices

$$
\begin{aligned}
& i_{B}\left(Y_{0}^{k}\right)-i_{B}\left(Y_{1}^{k}\right)=(-1)^{\sigma(1)} v_{1}+(-1)^{\sigma(2)} v_{2}+\cdots+(-1)^{\sigma(j-1)} v_{j-1}+ \\
&(-1)^{\sigma(j)+1} v_{j}+(-1)^{\sigma(j+1)+1} v_{j+1}+\cdots+(-1)^{\sigma(k-1)+1} v_{k-1} \\
&=(-1)^{\mu(1)} v_{1}+(-1)^{\mu(2)} v_{2}+\cdots+(-1)^{\mu(k-1)} v_{k-1},
\end{aligned}
$$

where

$$
\mu(\ell) \equiv_{2} \begin{cases}\sigma(\ell), & \ell \leq j-1 \\ \sigma(\ell)+1, & \ell \geq j\end{cases}
$$

as desired.

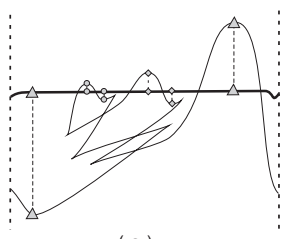

(a)

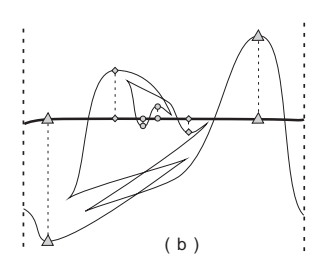

(b)

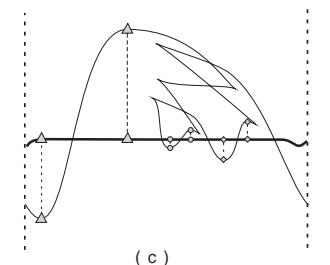

(c)

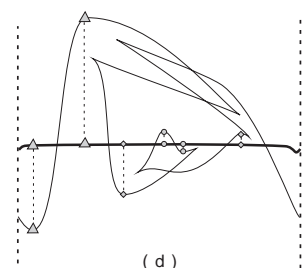

(d)

Figure 6.2.1: Links that differ by horizontal flypes: (a) $(2,1,2,2,2)$, (b) $\left(2,1,2^{1}, 2,2\right)$, (c) $\left(2,1,2,2,2^{1}\right),(\mathrm{d})\left(2,1,2^{1}, 2,2^{1}\right)$. If $L$ and $L^{\prime}$ differ by a horizontal flype from the $j^{\text {th }}$ component, then there will be a change in the indices of the critical points associated to the terms $h_{j+1}, \ldots, h_{n}$.

Remark 6.3 Applying vertical flypes to a link will leave the positive and negative homology polynomials unchanged. This follows since if

$$
\begin{aligned}
L & =\left(2 h_{n}, v_{n-1}^{q_{n-1}}, 2 h_{h-1}^{p_{n-1}}, \ldots, v_{1}^{q_{1}}, 2 h_{1}^{p_{1}}\right), \\
L^{\prime} & =\left(2 h_{n}, v_{n-1}^{w_{n-1}}, 2 h_{h-1}^{p_{n-1}}, \ldots, v_{1}^{w_{1}}, 2 h_{1}^{p_{1}}\right),
\end{aligned}
$$


where for some $j, w_{k}=q_{k}$, for $k \neq j$, and $w_{j}=q_{j}+1$, then there exist difference functions $\Delta$ and $\Delta^{\prime}$ for $L$ and $L^{\prime}$ with the same critical values and the same shifted indices. Similar to the situation in the proof of Theorem 6.2, a vertical flype from the $j^{\text {th }}$ component will affect the indices of the branches that contain points of the pairs associated to the terms $2 h_{j+1}, \ldots, 2 h_{n}$. Although the branch index associated to each point in the pair will change, the difference between the branch indices of this pair is unchanged.

Theorem 6.4 Consider the legendrian links

$L_{1}=\left(2 h_{n}, v_{n-1}, 2 h_{n-1}^{p_{n-1}}, \ldots, v_{1}, 2 h_{1}^{p_{1}}\right), L_{2}=\left(2 k_{m}, u_{m-1}, 2 k_{m-1}^{w_{m-1}}, \ldots, u_{1}, 2 k_{1}^{w_{1}}\right)$.

Then

$$
\begin{aligned}
& \Gamma^{-}(\lambda)\left[L_{1} \# L_{2}\right]=\Gamma^{-}(\lambda)\left[L_{1}\right]+\Gamma^{-}(\lambda)\left[L_{2}\right] ; \\
& \Gamma^{+}(\lambda)\left[L_{1} \# L_{2}\right]= \begin{cases}\Gamma^{+}(\lambda)\left[L_{1}\right]+\Gamma^{+}(\lambda)\left[L_{2}\right], & h_{1}, k_{1} \geq 1 \\
\Gamma^{+}(\lambda)\left[L_{1}\right]+\Gamma^{+}(\lambda)\left[L_{2}\right]-(1+\lambda), & \text { else. }\end{cases}
\end{aligned}
$$

Proof Let : $L_{i}$ : denote the tangles whose closure give $L_{i}$. It is possible to isotop the tangles : $L_{1}:$ and $: L_{2}:$ to the configurations similar to those used to calculate $\Gamma^{+}(\lambda)\left[L_{i}\right]$ and $\Gamma^{-}(\lambda)\left[L_{i}\right]$ but "scaled" so there exists an $M \in \mathbb{N}$ such that all critical values associated to : $L_{1}$ : are contained in $[-M, M]$, while all critical values associated to : $L_{2}$ : are contained in $(-\infty,-M) \cup(M, \infty)$. Figure 6.4.1 illustrates this construction when $p_{1}=0=w_{1}$.

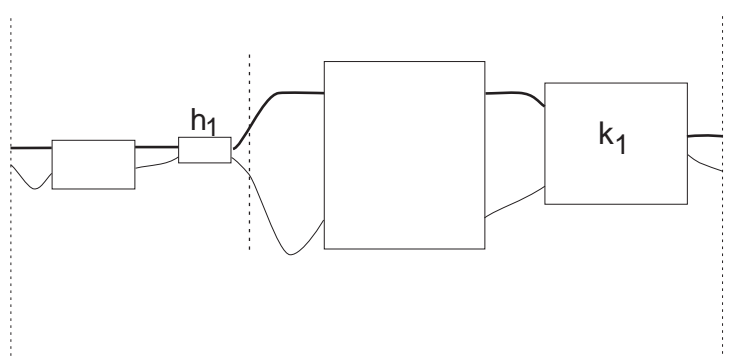

Figure 6.4.1: The connect sum of $L_{1}$ and $L_{2}$, when $p_{1}=w_{1}=0$, conveniently scaled.

When $h_{1}, k_{1} \geq 1$, this procedure gives rise to a difference function $\Delta$ with $2\left(h_{1}+\cdots+h_{n}+k_{1}+\cdots+k_{m}\right)$ critical points: for $j=1, \ldots, n, \iota=1, \ldots, m$, $\Delta$ has $h_{j}$ critical points of shifted index $i_{j}+1$ and value $c_{j}^{+}, h_{j}$ critical points 
of shifted index $i_{j}$ and value $c_{j}^{-}, k_{\iota}$ critical points of shifted index $\ell_{\iota}+1$ and value $d_{\iota}^{+}$, and $k_{\iota}$ critical points of shifted index $\ell_{\iota}$ and value $d_{\iota}^{-}$, where

$$
\begin{aligned}
d_{1}^{-}<d_{2}^{-}<\cdots<d_{m}^{-}<c_{1}^{-}<c_{2}^{-}<\cdots<c_{n}^{-}<0 \\
0<c_{n}^{+}<\cdots<c_{2}^{+}<c_{1}^{+}<d_{m}^{+}<\cdots<d_{2}^{+}<d_{1}^{+} .
\end{aligned}
$$

Arguments as in the proofs of Theorems 6.1 and 6.2 show that the hypotheses of Proposition 4.2 are satisfied. Thus

$$
\begin{aligned}
& \Gamma^{-}(\lambda)\left[L_{1} \# L_{2}\right]=\sum_{j=1}^{n} h_{j} \lambda^{i_{j}}+\sum_{\iota=1}^{m} k_{\iota} \lambda^{\ell_{\iota}}=\Gamma^{-}(\lambda)\left[L_{1}\right]+\Gamma^{-}(\lambda)\left[L_{2}\right], \\
& \Gamma^{+}(\lambda)\left[L_{1} \# L_{2}\right]=\sum_{j=1}^{n} h_{j} \lambda^{i_{j}+1}+\sum_{\iota=1}^{m} k_{\iota} \lambda^{\ell_{\iota}+1}=\Gamma^{+}(\lambda)\left[L_{1}\right]+\Gamma^{+}(\lambda)\left[L_{2}\right] .
\end{aligned}
$$

For the case $h_{1}=0$ or $k_{1}=0$, since $L_{1} \# L_{2}$ is equivalent to $L_{2} \# L_{1}$, it can be assumed $h_{1}=0$. In this case, two positive critical points that were necessary so that the tangles : $L_{i}$ : closed to links can be eliminated. Once $L_{1} \# L_{2}$ is positioned so that these points are eliminated, it is possible to construct a $\Delta$ that satisfies all the hypotheses of either Proposition 4.2 or Proposition 4.3. More precisely, when $k_{1} \geq 1$, it is possible to legendrian isotop the link $L_{1} \# L_{2}$ to a position so that it has a generating function $\Delta$ with $2\left(h_{2}+\cdots+h_{n}+\right.$ $\left.k_{1}+\cdots+k_{m}\right)$ critical points: for $j=2, \ldots, n, \iota=1, \ldots, m, \Delta$ has $h_{j}$ critical points of shifted index $i_{j}+1$ and value $c_{j}^{+}, h_{j}$ critical points of shifted index $i_{j}$ and value $c_{j}^{-}, k_{\iota}$ critical points of shifted index $\ell_{\iota}+1$ and value $d_{\iota}^{+}$, and $k_{\iota}$ critical points of shifted index $\ell_{\iota}$ and value $d_{\iota}^{-}$, where

$$
\begin{aligned}
d_{1}^{-}<d_{2}^{-}<\cdots<d_{m}^{-}<c_{2}^{-}< & \cdots<c_{n}^{-}<0 \\
& 0<c_{n}^{+}<\cdots<c_{2}^{+}<d_{m}^{+}<\cdots<d_{2}^{+}<d_{1}^{+} .
\end{aligned}
$$

All hypotheses of Proposition 4.2 are satisfied, and thus

$$
\begin{aligned}
\Gamma^{-}(\lambda)\left[L_{1} \# L_{2}\right] & =\sum_{j=2}^{n} h_{j} \lambda^{i_{j}}+\sum_{\iota=1}^{m} k_{\iota} \lambda^{\ell_{\iota}}=\Gamma^{-}(\lambda)\left[L_{1}\right]+\Gamma^{-}(\lambda)\left[L_{2}\right], \\
\Gamma^{+}(\lambda)\left[L_{1} \# L_{2}\right] & =\sum_{j=2}^{n} h_{j} \lambda^{i_{j}+1}+\sum_{\iota=1}^{m} k_{\iota} \lambda^{\ell_{\iota}+1} \\
& =\left(\Gamma^{+}(\lambda)\left[L_{1}\right]-(1+\lambda)\right)+\Gamma^{+}(\lambda)\left[L_{2}\right] .
\end{aligned}
$$

When $k_{1}=0$, it is possible to legendrian isotop the link $L_{1} \# L_{2}$ to a position so that it has a generating function $\Delta$ with $2\left(h_{2}+\cdots+h_{n}+k_{2}+\cdots+k_{m}\right)+2$ critical points: $\Delta$ has 1 critical point of shifted index 0 with value $d_{1}^{0}, 1$ critical point of shifted index 1 with value $d_{1}^{1}$, for $j=2, \ldots, n, \iota=2, \ldots, m, \Delta$ has $h_{j}$ 
critical points of shifted index $i_{j}+1$ and value $c_{j}^{+}, h_{j}$ critical points of shifted index $i_{j}$ and value $c_{j}^{-}, k_{\iota}$ critical points of shifted index $\ell_{\iota}+1$ and value $d_{\iota}^{+}$, and $k_{\iota}$ critical points of shifted index $\ell_{\iota}$ and value $d_{\iota}^{-}$, where

$$
\begin{aligned}
d_{2}^{-}<\cdots<d_{m}^{-}<c_{2}^{-}< & \cdots<c_{n}^{-}<0 \\
& 0<c_{n}^{+}<\cdots<c_{2}^{+}<d_{m}^{+}<\cdots<d_{2}^{+}<d_{1}^{0}<d_{1}^{1} .
\end{aligned}
$$

All hypotheses of Proposition 4.3 are satisfied, and thus

$$
\begin{aligned}
& \Gamma^{-}(\lambda)\left[L_{1} \# L_{2}\right]= \sum_{j=2}^{n} h_{j} \lambda^{i_{j}}+\sum_{\iota=2}^{m} k_{\iota} \lambda^{\ell_{\iota}}=\Gamma^{-}(\lambda)\left[L_{1}\right]+\Gamma^{-}(\lambda)\left[L_{2}\right], \\
& \Gamma^{+}(\lambda)\left[L_{1} \# L_{2}\right]=(1+\lambda)+\sum_{j=2}^{n} h_{j} \lambda^{i_{j}+1}+\sum_{\iota=2}^{m} k_{\iota} \lambda^{\ell_{\iota}+1}=\Gamma^{+}(\lambda)\left[L_{1}\right] \\
&+\left(\Gamma^{+}(\lambda)\left[L_{2}\right]-(1+\lambda)\right) .
\end{aligned}
$$

\section{Applications}

In this section, the polynomial calculations of Section 6 will be applied to show that "most" of the legendrian links $L_{n}=\left(2 h_{n}, v_{n-1}, \ldots, 2 h_{1}\right)$ are ordered. In addition, by analyzing the polynomials resulting from the swap and the flype operations, lower bounds will be given for the number of different minimal legendrian representations of a given topological link type.

The following proposition shows that there is a simple relation between the polynomials of a rational link and its swap.

Proposition 7.1 Let $L=\left(2 h_{n}, v_{n-1}, 2 h_{n-2}^{p_{n-2}}, \ldots, h_{2}^{p_{2}}, v_{1}, 2 h_{1}^{p_{1}}\right)$, and let $\bar{L}$ denote the swap of $L$. Then

$$
\Gamma^{-}(\lambda)[\bar{L}]= \begin{cases}\overline{\Gamma^{-}(\lambda)[L]}, & h_{1} \geq 1, \\ (1+\lambda)+\overline{\Gamma^{-}(\lambda)[L]}, & h_{1}=0,\end{cases}
$$

where $\overline{\Gamma^{-}(\lambda)[L]}$ denotes the palindrome of $\Gamma^{-}(\lambda)[L]$.

Proof This follows easily from Theorem 3.16 and Theorem 6.2. For $h_{1} \geq 1$,

$$
\lambda \cdot \Gamma^{-}(\lambda)[L]=\Gamma^{+}(\lambda)[L]=\lambda \cdot \overline{\Gamma^{-}(\lambda)[\bar{L}]} .
$$

For $h_{1}=0$,

$$
(1+\lambda)+\lambda \cdot \Gamma^{-}(\lambda)[L]=\Gamma^{+}(\lambda)[L]=\lambda \cdot \overline{\Gamma^{-}(\lambda)[\bar{L}]} .
$$

This implies, by examining palindromes, that

$$
\lambda^{-1} \Gamma^{-}(\lambda)[\bar{L}]=1+\lambda^{-1}+\lambda^{-1} \overline{\Gamma^{-}(\lambda)[L]},
$$

and the desired result follows. 
Theorem 7.2 Consider the legendrian link $L=\left(2 h_{n}, v_{n-1}, 2 h_{n-1}, \ldots, v_{1}\right.$, $\left.2 h_{1}\right)$. If $h_{1} \geq 1$, then $L$ is ordered iff $L \neq\left(2 h_{1}\right)$. If $h_{1}=0$, then $L$ is ordered.

Proof First suppose that $h_{1} \geq 1$. When $L=\left(2 h_{1}\right)$, it is easy to explicitly check that $L$ is not ordered. Suppose $L=\left(2 h_{n}, v_{n-1}, \ldots, v_{1}, 2 h_{1}\right), n \geq 2$. By Proposition 7.1, to show $L$ is ordered, it suffices to prove that $\Gamma^{-}(\lambda)[L]$ is not palindromic. By Theorem 6.1,

$$
\Gamma^{-}(\lambda)[L]=h_{1}+h_{2} \lambda^{-v_{1}}+h_{3} \lambda^{-v_{1}-v_{2}}+\cdots+h_{n} \lambda^{-v_{1}-v_{2}-\cdots-v_{n-1}},
$$

and, since $n \geq 2, \Gamma^{-}(\lambda)[L]$ is not palindromic.

Next suppose $h_{1}=0$. By Proposition 7.1, it suffices to verify that $(1+\lambda)+$ $\lambda \cdot \Gamma^{-}(\lambda)[L] \neq \lambda \cdot \overline{\Gamma^{-}(\lambda)[L]}$. By Theorem 6.1,

$$
\lambda \cdot \overline{\Gamma^{-}(\lambda)[L]}=\sum_{m=-\infty}^{\infty} a_{m} \lambda^{m}, \quad \text { with } a_{1}=0,
$$

while $\lambda \cdot \Gamma^{-}(\lambda)[L]=\sum_{m=-\infty}^{\infty} b_{m} t^{m}$, with $b_{1}=0$, and thus

$$
(1+\lambda)+\lambda \cdot \Gamma^{-}(\lambda)[L]=\sum_{m=-\infty}^{\infty} c_{m} \lambda^{m}, \quad \text { with } c_{1}=1 .
$$

Thus $L$ must be ordered.

Distinct minimal legendrian versions of the topological link

$$
L_{n}=\left(2 h_{n}, v_{n-1}, 2 h_{n-1} \ldots, 2 h_{2}, v_{1}, 2 h_{1}\right), \quad h_{n}, \ldots, v_{1} \geq 1, h_{1} \geq 1,
$$

will now be enumerated. For $h_{1} \geq 1$, there are potentially $2 \cdot 2^{n-1}$ legendrian versions arising from the swap and the flype operations that can be distinguished by the polynomials. However, the following proposition shows that the polynomials cannot distinguish all these swaps and flypes: the swap operation always produces a link with the same polynomials as some flype.

Proposition 7.3 For $h_{1} \geq 1, p_{1} \in\left\{0, \ldots, 2 h_{1}-1\right\}$, and $p_{i} \in\left\{0, \ldots, 2 h_{i}\right\}$ when $i \geq 2$, consider the minimal legendrian links

$$
\begin{aligned}
& L_{1}=\left(2 h_{n}, v_{n-1}, 2 h_{n-1}^{p_{n-1}}, \ldots, 2 h_{2}^{p_{2}}, v_{1}, 2 h_{1}^{p_{1}}\right), \\
& L_{2}=\left(2 h_{n}, v_{n-1}, 2 h_{n-1}^{p_{n-1}}, \ldots, 2 h_{2}^{p_{2}}, v_{1}, 2 h_{1}^{p_{1}+1}\right),
\end{aligned}
$$

Let $\overline{L_{1}}$ be the swap of $L_{1}$. Then $\Gamma^{-}(\lambda)\left[\overline{L_{1}}\right]=\Gamma^{-}(\lambda)\left[L_{2}\right]$, and $\Gamma^{+}(\lambda)\left[\overline{L_{1}}\right]=$ $\Gamma^{+}(\lambda)\left[L_{2}\right]$. 
Proof It suffices to prove that $\Gamma^{-}(\lambda)\left[\overline{L_{1}}\right]=\Gamma^{-}(\lambda)\left[L_{2}\right]$. By Proposition 7.1 and Theorem 6.2 , for $\sigma(j)=1+\sum_{i=1}^{j} p_{i} \bmod 2$,

$$
\begin{aligned}
\Gamma^{-}(\lambda)\left[\overline{L_{1}}\right] & =\overline{\Gamma^{-}(\lambda)\left[L_{1}\right]}=\overline{h_{1}+\sum_{i=2}^{n} h_{i} \lambda^{(-1)^{\sigma(1)} v_{1}+(-1)^{\sigma(2)} v_{2}+\cdots+(-1)^{\sigma(i-1)} v_{i-1}}} \\
& =h_{1}+\sum_{i=2}^{n} h_{i} \lambda^{(-1)^{\sigma(1)+1} v_{1}+(-1)^{\sigma(2)+1} v_{2}+\cdots+(-1)^{\sigma(i-1)+1} v_{i-1}} \\
& =\Gamma^{-}(\lambda)\left[L_{2}\right] .
\end{aligned}
$$

Thus the swap and flype operations give at most $2^{n-1}$ legendrian versions of $L_{n}$ that can be distinguished by the polynomials. In fact, for $n=3$, there are often at least $4=2^{3-1}$ versions of $L_{3}$ distinguishable by the polynomials.

Theorem 7.4 Consider the topological link

$$
L_{3}=\left(2 h_{3}, v_{2}, 2 h_{2}, v_{1}, 2 h_{1}\right), \quad h_{3}, h_{2}, v_{2}, v_{1} \geq 1, \quad h_{1} \geq 0 .
$$

If $h_{1}=0$, then there are at least 4 minimal legendrian versions of $L_{3}$. If $h_{1} \geq 1$ and either $v_{2} \neq 2 v_{1}$ or $h_{2} \neq h_{3}$, then there are at least 4 minimal legendrian versions of $L_{3}$.

Proof For $h_{1}=0$, consider

$$
\begin{array}{ll}
L_{3}^{0}=\left(2 h_{3}, v_{2}, 2 h_{2}^{0}, v_{1}, 0\right), & L_{3}^{1}=\overline{\left(2 h_{3}, v_{2}, 2 h_{2}^{1}, v_{1}, 0\right),} \\
L_{3}^{2}=\overline{\left(2 h_{3}, v_{2}, 2 h_{2}^{0}, v_{1}, 0\right),} & L_{3}^{3}=\overline{\left(2 h_{3}, v_{2}, 2 h_{2}^{1}, v_{1}, 0\right) .}
\end{array}
$$

By Theorem 6.2 and Proposition 7.1,

$$
\begin{gathered}
\Gamma^{-}(\lambda)\left[L_{3}^{0}\right]=h_{2} \lambda^{-v_{1}}+h_{3} \lambda^{-v_{1}-v_{2}}, \quad \Gamma^{-}(\lambda)\left[L_{3}^{1}\right]=h_{2} \lambda^{-v_{1}}+h_{3} \lambda^{-v_{1}+v_{2}}, \\
\Gamma^{-}(\lambda)\left[L_{3}^{2}\right]=1+\lambda+h_{2} \lambda^{+v_{1}}+h_{3} \lambda^{+v_{1}+v_{2}}, \\
\Gamma^{-}(\lambda)\left[L_{3}^{3}\right]=1+\lambda+h_{2} \lambda^{+v_{1}}+h_{3} \lambda^{+v_{1}-v_{2}} .
\end{gathered}
$$

It is easy to verify that for all choices of $h_{i}, v_{i} \geq 1$, these must be distinct polynomials, and thus $L_{3}^{i}, i=0, \ldots 3$, are legendrian distinct.

For $h_{1} \geq 1$, consider

$$
\begin{array}{ll}
L_{3}^{(0,0)}=\left(2 h_{3}, v_{2}, 2 h_{2}^{0}, v_{1}, 2 h_{1}^{0}\right), & L_{3}^{(1,0)}=\left(2 h_{3}, v_{2}, 2 h_{2}^{1}, v_{1}, 2 h_{1}^{0}\right), \\
L_{3}^{(0,1)}=\left(2 h_{3}, v_{2}, 2 h_{2}^{0}, v_{1}, 2 h_{1}^{1}\right), & L_{3}^{(1,1)}=\left(2 h_{3}, v_{2}, 2 h_{2}^{1}, v_{1}, 2 h_{1}^{1}\right) .
\end{array}
$$


By Theorem 6.2,

$$
\begin{aligned}
& \Gamma^{-}(\lambda)\left[L_{3}^{(0,0)}\right]=h_{1}+h_{2} \lambda^{-v_{1}}+h_{3} \lambda^{-v_{1}-v_{2}}, \\
& \Gamma^{-}(\lambda)\left[L_{3}^{(1,0)}\right]=h_{1}+h_{2} \lambda^{-v_{1}}+h_{3} \lambda^{-v_{1}+v_{2}}, \\
& \Gamma^{-}(\lambda)\left[L_{3}^{(0,1)}\right]=h_{1}+h_{2} \lambda^{+v_{1}}+h_{3} \lambda^{+v_{1}+v_{2}} \\
& \Gamma^{-}(\lambda)\left[L_{3}^{(1,1)}\right]=h_{1}+h_{2} \lambda^{+v_{1}}+h_{3} \lambda^{+v_{1}-v_{2}} .
\end{aligned}
$$

The condition $v_{2} \neq 2 v_{1}$ or $h_{2} \neq h_{3}$ implies that all these polynomials are distinct. Thus there are at least 4 distinct legendrian versions of $L_{3}$.

The following condition on $h_{i}$ will guarantee that all the flypes have distinct $\Gamma^{-}(\lambda)$ polynomials. Such sets arise in Additive Number Theory; see [12].

Definition 7.5 A set $\left\{h_{1}, \ldots, h_{n}\right\}$ of integers is said to have distinct subset sums if the sums of all its $2^{n}$ subsets are distinct. Such a set will be abbreviated as a d.s.s. set.

It is easy to verify that $\{1,2\},\{1,2,4\}$ and $\{2,3,4,8\}$ are d.s.s. sets, while $\{1,2,3\}$ is not. In general, $\left\{2^{i}: 0 \leq i \leq k\right\}$ is a d.s.s. set of order $k+1$. There is an Erdös prize associated to finding the largest order of a d.s.s. set with entries positive and bounded above by $2^{k}$.

Theorem 7.6 For $n \geq 4$, consider the topological link

$$
L_{n}=\left(2 h_{n}, v_{n-1}, 2 h_{n-1}^{0}, \ldots, 2 h_{2}^{0}, v_{1}, 2 h_{1}^{0}\right), \quad h_{n}, v_{n-1}, \ldots, v_{1} \geq 1, h_{1} \geq 0 .
$$

If $h_{1} \geq 1$, assume $\left\{h_{1}, h_{2}, \ldots, h_{n}\right\}$ form a d.s.s. set of order $n$, while if $h_{1}=0$, assume $\left\{1, h_{2}, \ldots, h_{n}\right\}$ form a d.s.s. set of order $n$. Then there exist at least $2^{n-1}$ legendrian versions of $L_{n}$.

Proof For the case where $h_{1} \geq 1$, consider the links

$$
\left(2 h_{n}, v_{n-1}, 2 h_{n-1}^{p_{n-1}}, \ldots, 2 h_{2}^{p_{2}}, v_{1}, 2 h_{1}^{p_{1}}\right), \quad p_{n-1}, \ldots, p_{1} \in \mathbb{Z}_{2} .
$$

It will be shown that distinct choices of $\left(p_{n-1}, \ldots, p_{1}\right) \in \mathbb{Z}_{2} \times \cdots \times \mathbb{Z}_{2}$ give rise to legendrian links with distinct $\Gamma^{-}(\lambda)$ polynomials. Let

$$
\begin{aligned}
& L_{1}=\left(2 h_{n}, v_{n-1}, 2 h_{n-1}^{p_{n-1}}, \ldots, 2 h_{2}^{p_{2}}, v_{1}, 2 h_{1}^{p_{1}}\right), \\
& L_{2}=\left(2 h_{n}, v_{n-1}, 2 h_{n-1}^{q_{n-1}}, \ldots, 2 h_{2}^{q_{2}}, v_{1}, 2 h_{1}^{q_{1}}\right)
\end{aligned}
$$

and suppose that $\Gamma^{-}(\lambda)\left[L_{1}\right]=\Gamma^{-}(\lambda)\left[L_{2}\right]$. By Theorem 6.2,

$\Gamma^{-}(\lambda)\left[L_{1}\right]=h_{1}+h_{2} \lambda^{s_{2}}+\cdots+h_{n} \lambda^{s_{n}}, \quad \Gamma^{-}(\lambda)\left[L_{2}\right]=h_{1}+h_{2} \lambda^{m_{2}}+\cdots+h_{n} \lambda^{m_{n}}$ 
for

$$
\begin{aligned}
s_{k} & =(-1)^{\sigma(1)} v_{1}+(-1)^{\sigma(2)} v_{2}+\cdots+(-1)^{\sigma(k-1)} v_{k-1}, \\
m_{k} & =(-1)^{\mu(1)} v_{1}+(-1)^{\mu(2)} v_{2}+\cdots+(-1)^{\mu(n-1)} v_{n-1}, \quad k=2, \ldots, n,
\end{aligned}
$$

where $\sigma(s)=1+\sum_{t=1}^{s} p_{t} \bmod 2, \mu(s)=1+\sum_{t=1}^{s} q_{t} \bmod 2$. If it is shown that $s_{k}=m_{k}, \forall k$, then $\sigma(k)=\mu(k), \forall k$, and thus $p_{k}=q_{k}, \forall k$.

First $\Gamma^{-}(\lambda)\left[L_{1}\right]$ and $\Gamma^{-}(\lambda)\left[L_{2}\right]$ will be rewritten in terms of distinct powers of $\lambda$. Choose $I_{0}, \ldots, I_{N}$ with $I_{0}<\cdots<I_{N}$ to be the distinct elements of $\left\{0, s_{2}, \ldots, s_{n}\right\}=\left\{0, m_{2}, \ldots, m_{n}\right\}$. Then

$$
\Gamma^{-}(\lambda)\left[L_{1}\right]=\sum_{\alpha=0}^{N} \sum_{k=1}^{K_{\alpha}} h_{i_{k}^{\alpha}} \lambda^{I_{\alpha}}, \quad \Gamma^{-}(\lambda)\left[L_{2}\right]=\sum_{\alpha=0}^{N} \sum_{k=1}^{L_{\alpha}} h_{j_{k}^{\alpha}} \lambda^{I_{\alpha}},
$$

where, for all $\alpha, h_{i_{1}^{\alpha}}<h_{i_{2}^{\alpha}}<\cdots<h_{i_{K_{\alpha}}^{\alpha}}$, and $h_{j_{1}^{\alpha}}<h_{j_{2}^{\alpha}}<\cdots<h_{j_{L_{\alpha}}^{\alpha}}$. Since $\left\{h_{1}, \ldots, h_{n}\right\}$ are a d.s.s. set, $\Gamma^{-}(\lambda)\left[L_{1}\right]=\Gamma^{-}(\lambda)\left[L_{2}\right]$ implies $K_{\alpha}=L_{\alpha}$, for all $\alpha$, and that $i_{k}^{\alpha}=j_{k}^{\alpha}$, for all $\alpha$ and $k$. This implies $s_{k}=m_{k}$, as desired.

For the case where $h_{1}=0$, consider the links

$$
\left(2 h_{n}, v_{n-1}, 2 h_{n-1}^{p_{n-1}}, \ldots, 2 h_{2}^{p_{2}}, v_{1}, 0\right), \quad \overline{\left(2 h_{n}, v_{n-1}, 2 h_{n-1}^{p_{n-1}}, \ldots, 2 h_{2}^{p_{2}}, v_{1}, 0\right)},
$$

where $p_{n-1}, \ldots, p_{2} \in \mathbb{Z}_{2}$. It will be shown that the $2^{n-2}$ choices of $\left(p_{n-1}, \ldots, p_{2}\right)$ $\in \mathbb{Z}_{2} \times \cdots \times \mathbb{Z}_{2}$ give rise to $2 \cdot 2^{n-2}$ legendrian links with distinct $\Gamma^{-}(\lambda)$ polynomials. By Theorem 6.2 and Propositions 7.1 and 7.3,

$$
\begin{aligned}
& \Gamma^{-}(\lambda)\left[\left(2 h_{n}, v_{n-1}, 2 h_{n-1}^{p_{n-1}}, \ldots, 2 h_{2}^{p_{2}}, v_{1}, 0\right)\right] \\
& =\Gamma^{-}(\lambda)\left[\left(2 h_{n}, v_{n-1}, 2 h_{n-1}^{p_{n-1}}, \ldots, 2 h_{2}^{p_{2}}, v_{1}, 2^{0}\right)\right]-1, \\
& \Gamma^{-}(\lambda)\left[\overline{\left(2 h_{n}, v_{n-1}, 2 h_{n-1}^{p_{n-1}}, \ldots, 2 h_{2}^{p_{2}}, v_{1}, 0\right)}\right] \\
& =\overline{\Gamma^{-}(\lambda)\left[\left(2 h_{n}, v_{n-1}, 2 h_{n-1}^{p_{n-1}}, \ldots, 2 h_{2}^{p_{2}}, v_{1}, 0\right)\right]}+1+\lambda \\
& =\overline{\Gamma^{-}(\lambda)\left[\left(2 h_{n}, v_{n-1}, 2 h_{n-1}^{p_{n-1}}, \ldots, 2 h_{2}^{p_{2}}, v_{1}, 2^{0}\right)\right]-1}+1+\lambda \\
& =\overline{\Gamma^{-}(\lambda)\left[\left(2 h_{n}, v_{n-1}, 2 h_{n-1}^{p_{n-1}}, \ldots, 2 h_{2}^{p_{2}}, v_{1}, 2^{0}\right)\right]}+\lambda \\
& =\Gamma^{-}(\lambda)\left[\left(2 h_{n}, v_{n-1}, 2 h_{n-1}^{p_{n-1}}, \ldots, 2 h_{2}^{p_{2}}, v_{1}, 2^{1}\right)\right]+\lambda .
\end{aligned}
$$

Thus to show that these $2^{n-1}$ polynomials are distinct, the following three statements will be proven.

(1) If $\left(p_{n-1}, \ldots, p_{2}\right) \neq\left(q_{n-1}, \ldots, q_{2}\right)$, then

$$
\begin{aligned}
\Gamma^{-}(\lambda)\left[\left(2 h_{n}, v_{n-1}, 2 h_{n-1}^{p_{n-1}}, \ldots, 2 h_{2}^{p_{2}}, v_{1}, 2^{0}\right)\right]-1 \neq \\
\Gamma^{-}(\lambda)\left[\left(2 h_{n}, v_{n-1}, 2 h_{n-1}^{q_{n-1}}, \ldots, 2 h_{2}^{q_{2}}, v_{1}, 2^{0}\right)\right]-1 ;
\end{aligned}
$$


(2) If $\left(p_{n-1}, \ldots, p_{2}\right) \neq\left(q_{n-1}, \ldots, q_{2}\right)$, then

$$
\begin{aligned}
\Gamma^{-}(\lambda)\left[\left(2 h_{n}, v_{n-1}, 2 h_{n-1}^{p_{n-1}}, \ldots, 2 h_{2}^{p_{2}}, v_{1}, 2^{1}\right)\right]+\lambda \neq \\
\Gamma^{-}(\lambda)\left[\left(2 h_{n}, v_{n-1}, 2 h_{n-1}^{q_{n-1}}, \ldots, 2 h_{2}^{q_{2}}, v_{1}, 2^{1}\right)\right]+\lambda ;
\end{aligned}
$$

(3) For all $\left(p_{n-1}, \ldots, p_{2}\right),\left(q_{n-1}, \ldots, q_{2}\right)$,

$$
\begin{aligned}
\Gamma^{-}(\lambda)\left[\left(2 h_{n}, v_{n-1}, 2 h_{n-1}^{p_{n-1}}, \ldots, 2 h_{2}^{p_{2}}, v_{1}, 2^{0}\right)\right]-1 \neq \\
\Gamma^{-}(\lambda)\left[\left(2 h_{n}, v_{n-1}, 2 h_{n-1}^{q_{n-1}}, \ldots, 2 h_{2}^{q_{2}}, v_{1}, 2^{1}\right)\right]+\lambda .
\end{aligned}
$$

Statements (1) and (2) follow from Theorem 6.2. To verify statement (3), suppose that there exist $\left(p_{n-1}, \ldots, p_{2}\right),\left(q_{n-1}, \ldots, q_{2}\right)$ such that

$$
\begin{aligned}
& \Gamma^{-}(\lambda)\left[\left(2 h_{n}, v_{n-1}, 2 h_{n-1}^{p_{n-1}}, \ldots, 2 h_{2}^{p_{2}}, v_{1}, 2^{0}\right)\right]-1= \\
& \Gamma^{-}(\lambda)\left[\left(2 h_{n}, v_{n-1}, 2 h_{n-1}^{q_{n-1}}, \ldots, 2 h_{2}^{q_{2}}, v_{1}, 2^{1}\right)\right]+\lambda .
\end{aligned}
$$

By writing the polynomials in terms of distinct powers of $\lambda$,

$$
\begin{aligned}
& \Gamma^{-}(\lambda)\left[\left(2 h_{n}, v_{n-1}, 2 h_{n-1}^{p_{n-1}}, \ldots, 2 h_{2}^{p_{2}}, v_{1}, 2^{0}\right)\right]-1=\sum a_{i} t^{i}, \\
& \quad \text { where } a_{0}=h_{i_{1}}+\cdots+h_{i_{K}}, \quad h_{i_{1}}, \ldots, h_{i_{K}} \in\left\{h_{2}, \ldots, h_{n}\right\},
\end{aligned}
$$

while

$$
\begin{aligned}
& \Gamma^{-}(\lambda)\left[\left(2 h_{n}, v_{n-1}, 2 h_{n-1}^{q_{n-1}}, \ldots, 2 h_{2}^{q_{2}}, v_{1}, 2^{1}\right)\right]+\lambda=\sum b_{i} \lambda^{i}, \\
& \quad \text { where } b_{0}=1+h_{j_{1}}+\cdots+h_{j_{L}}, \quad h_{j_{1}}, \ldots, h_{j_{L}} \in\left\{h_{2}, \ldots, h_{n}\right\} .
\end{aligned}
$$

The assumption that these polynomials are equal contradicts the hypothesis that $\left\{1, h_{2}, \ldots, h_{n}\right\}$ are a d.s.s. set of order $n$. Thus statement (3) is true, and it follows that the $2^{n-1}$ polynomials are distinct.

\section{Acknowledgements}

During the preparation of this paper, I had the pleasure of visiting the American Institute of Mathematics in Palo Alto, CA for their program on contact geometry, and the Institute for Advanced Study in Princeton, NJ. I thank both of these institutions for their wonderful hospitality. I also thank my reviewers for numerous useful comments and suggestions.

This research was supported in part by NSF grant DMS 9971374 and, while visiting IAS, NSF grant DMS 97-29992. 


\section{References}

[1] C Adams, The Knot Book, W H Freeman and Company (1994)

[2] D Bennequin, Entrelacements et équation de Pfaff, Astérique 107-108 (1983) 87-162

[3] M Chaperon, On generating families, from: "The Floer Memorial Volume", (H Hofer, C Taubes, A Weinstein, E Zehnder, Editors) Birkhäuser (1995)

[4] Yu Chekanov, Critical points of quasifunctions, and generating families of legendrian manifolds, Funktsional.-Anal.-i Prilozhen (in Russian) 30 (1996) 5669

[5] Yu Chekanov, Differential algebras of Legendrian links, Fields Institute preprint FI-ST1997-010 (1997)

[6] J H Conway, An enumeration of knots and links, and some of their algebraic properties, from: "Computational problems in Abstract Algebra", Proc. Conf. Oxford, 1967 (J Leech, Editor) Pergamon Press New York (1970)

[7] Y Eliashberg, Legendrian and transversal knots in tight contact 3-manifolds, from: "Topological Methods in Modern Mathematics", (L R Goldberg and A V Phillips, Editors) Publish or Perish, Inc. (1993)

[8] Y Eliashberg, Invariants in contact topology, Doc. Math. J. DMV, Extra Volume ICM 1998 (electronic), 327-338

[9] Y Eliashberg, M Gromov, Lagrangian intersections theory: finite-dimensional approach, from: "Geometry of differential equations", Amer. Math. Soc. Transl. Ser. 2, 186 Amer. Math. Soc. Providence, RI (1998)

[10] Y Eliashberg, A Givental, H Hofer, Symplectic Field Theory, e-print arXiv:math.SG/0010059

[11] E Ferrand, On Legendrian knots and polynomial invariants, e-print arXiv: math.GT/0002250

[12] R Guy, Unsolved Problems in Number Theory, Springer-Verlag (1991)

[13] L Ng, Invariants of Legendrian Links, MIT Ph.D. Thesis (2001)

[14] L Ng, L Traynor, in preparation

[15] S Tabachnikov, Estimates for the Bennequin number of Legendrian links from state models for knot polynomials, Math. Res. Lett. 4 (1997) 143-156

[16] D Théret, A complete proof of Viterbo's uniqueness theorem on generating functions, Topology Appl. 96 249-266

[17] D Théret, A Lagrangian camel, Comm. Math. Helv. 74 (1999) 591-614

[18] L Traynor, Symplectic homology via generating functions, Geom. Funct. Anal. 4 (1994) 718-748

[19] L Traynor, Legendrian circular helix links, Math. Proc. Camb. Phil. Soc. 122 (1997) 301-314 
[20] L Traynor, A legendrian stratification of rational tangles, Journal of Knot Theory and its Ramifications 7 (1998) 659-700

[21] C Viterbo, Symplectic topology as the geometry of generating functions, Math. Ann. 292 (1992) 685-710

[22] C Viterbo, Generating Functions, Symplectic Geometry, and Applications, from: "Proceedings of the International Congress of Mathematicians, Zürich, Switzerland 1994", Birkhäuser-Verlag, Basel, Switzerland (1995) 537-547 